\title{
Studien über die Beeinflussung des Serumeiweisses und dessen kolloid=osmotischen Drucks durch perorale Zufuhr von grossen Wassermengen bei Gesunden sowie Hochdruck= und Nierenkranken.
}

\author{
von \\ Kuranosuke Shida. \\ (志田藏之助) \\ (Aus der Medizinischen Klinik von Prof. Dr. T. Kato, \\ Tohoku Reichsuniversität zu Sendai.)
}

Über die Weise, wie die Konzentration des Blutes dann geändert wird, wenn dem Organismus grosse Mengen Wassers per os zugeführt worden sind, ist bisher zur Erklärung des Wesens der Wasserdiurese von verschiedenen Seiten klinisch und experimentell untersucht worden. Diesbezügliche Versuchsresultate von früheren Autoren hatten sich unterschiedlich ergeben. So sahen manche Autoren (Danielu. Högler, ${ }^{1)}$ Hopmann u. Schüler, ${ }^{2)}$ Kobayashi, ${ }^{3)}$ GollwitzerMeier u. Rabl, ${ }^{4)}$ Brahn u. Bielschowsky, ${ }^{5)}$ Poulsson, ${ }^{6)}$ Klein u. Nonnenbruch, ${ }^{7)} \mathrm{Brucke}^{8)}$ u. a.) nach Wasserzufuhr eine Verdünnung des Blutes, während wieder andere (Reiss, ${ }^{9)} \mathrm{Veil},{ }^{10)} \mathrm{Halda}$ e u. Priestley, ${ }^{11)}$ Monakow, ${ }^{12)}$ Underhill u. Pick ${ }^{13)} u$. a.) hingegen eher eine Konzentrationszunahme konstatierten, und über diese Frage

\footnotetext{
1) Daniel a. Högler, Wien. Arch. f. inn. Med., 1922, 4, 167.

2) Hopmann u. S chüler, Ztschr. f. d. ges. exp. Med., 1922, 30, 148.

3) Ko bayas hi, Nissin Igaku, 1924, 14, 47.

4) Gollwitzer-Meier u. Rabl, Ztschr. f. d. ges. exp. Med., 1926, 53, 525.

5) Brahn u. Biels ch ow sky, Klin. Wsehr., 1928, 2004.

6) Poulss o n, Ztschr. f. d. ges. exp. Med., 1930, 71, 577.

7) Klein u. Nonnen bruch, Ztschr. f. klin. Med., 1930, 112, 568.

8) Brucke, Ztschr. f. klin. Med., 1932, 122, 164.

9) Rei s s, Ergebn. d. inn. Med. u. Kinderheilk., 1913, 10, 531.

10) Ve il, Dtsch. Arch. f. klin. Med., 1914, 113, 226.

11) Hald a ne u. Priestley, Journ. Physiol., 1916, 50, 296.

12) Mona kow, Dtsch. Arch. f. klin. Med., 1917, 122, 164.

13) Underhill u. Pick, Amer. Journ. Physiol,, 1923, 66, 520.
} 
war vielfach diskutiert worden. In der Folge aber ist durch grundlegende Untersuchungen von einer Reihe von Autoren, wie $\operatorname{Marx}{ }^{14}$ Wiechmann u. Liang, ${ }^{15)}$ Hitzenberger u. Merkler, ${ }^{16)} A$ be ${ }^{17)} u$. a. endgültig erkannt worden, dass die Schwankung der Blutkonzentration, die nach oraler Wasserzufuhr auftritt, einen Phasenverlauf nimmt, indem die Phase der Verdünnung und die der Konzentrierung abwechselnd in Erscheinung tritt.

In den Untersuchungen von oben genannten Autoren über die Blutveränderungen waren jedoch die Bestimmungen vorwiegend auf Hämoglobin, Erythrozytenzahl, Bluteiweiss und Salzgehalte eingeschränkt; die Untersuchungen aber, die sich mit physikalisch-chemischer Eigenschaft des Blutes, vor allem aber mit dem Verhalten des Bluteiweisses und dessen kolloid-osmotischen Drucks (k. o. D.), welche beide im innigsten Zusammenhang mit dem Wasseraustausch stehen, befassen, liegen sehr wenig vor; die Untersuchungen darüber sind bis dahin nur von $\mathrm{K}$ y lin, ${ }^{18)}$ Pelleg r in $\mathrm{i}^{19)}$ und Meyer ${ }^{20)}$ angestellt worden.

Kyl in ${ }^{18)}$ gab 5 Versuchspersonen je 1,000 ccm Wasser und fand bei 3 Fällen ein geringes Absinken von k. o. D. und eine Abnahme von k. o. D. pro 1\% Eiweiss (unten kurz als Druck pro \% bezeichnet), auch Pelle grin i ${ }^{19)}$ soll im grossen ganzen gleiches Ergebnis erhalten haben. Die Versuche von beiden Autoren sind aber an kleinem Krankenmaterial ausgeführt worden, mithin fehlt die Beweiskraft für den endgültigen Schluss. Auch M e y e $\mathrm{r}^{20)}$ hat an 9 Leichtkranken, welche anscheinend keine Störung im Wasserhaushalt aufwiesen, die orale Wasserzufuhr von $1,000 \mathrm{ccm}$ vorgenommen und die danach einsetzenden Schwankungen des k. o.D. des Blutes verfolgt; diese verhielten sich in bezug auf ihre Stärke, ihre Richtung und auch ihre Dauer individuell ganz verschieden, so dass er aus seinen Versuchsbefunden keine einheitliche Beziehung ausfindig machen konnte. Unter dem Krankheitsmaterial von $\mathrm{Me} \mathrm{yer}^{20)}$ gab es indes Fälle von abgelaufener Angina, leichtgradiger Pyelitis und auch mit postencephalitischem Zustand geringen Grades, welche alle nach derzeitigen Anschauungen der pathologischen Physiologie als nicht gerade unabhängig von irgendwelcher Störung des Wasserhaushaltes zu deuten sind. Der Grund für seine recht unterschiedlichen Ergebnisse dürfte also meines Erachtens darin begründet sein, dass er nicht auf diese Verhältnisse Rücksicht genommen hat.

14) M a r x, Dtsch. Arch. f. klin. Med., 1926, 153, 358.

15) Wiechmann u. Liang, Ibid., 1929, 163, 282.

16) Hitzen berger u. Merkler, Wien. Arch. f. inn. Med., 1930, $19,327$.

17) A b e, Tohoku Journ. Exp. Med., 1931, 17, 174.

18) Ky lin, Ztschr. f. "d. ges. exp. Med., 1929, 68, 746.

19) Pellegrini, Arch. Pat. e clin. Med., 1981, 10, 375. Ref. in Kongresszentralblatt f. d. ges. inn. Med., 1931, 63, 419.

20) Meyer, Ztschr. f. klin. Med., 1931, 116, 174, u. 117, 245. 
Nach dem oben Gesagten sind die Studien über die nach peroraler Zufuhr von grossen Mengen Wassers auftretende Veränderung des k. o. D. des Blutes noch nicht vollends abgeschlossen.

Über den Mechanismus der Wasserdiurese sind bis dahin zahlreiche Untersuchungen von vielen Autoren angestellt worden, ohne dass jedoch Übereinstimmung der Anschauungen erzielt werden konnte. Was das Wesen der Wasserdiurese anbelangt, sind verschiedene Auffassungen geäussert worden : Es wird nämlich angenommen, dass sie ausser durch die Nierenfunktion auch neuroendokrin gesteuert werden, oder die Leber hierbei die Hauptrolle spiele, es scheint ferner die Wasserdiurese in recht komplizierten Beziehungen zuZentren und Periphrie zu stehen. Angesichts dieser Sachlage dürfte die Analyse des Verhaltens des k. o. D. des Blutes allein nicht genügen, das Wesen der Wasserdiurese aufzuklären, wenn der k. o. D. des Blutes auch im Wasseraustausch eine wichtige Rolle spielt. Jedenfalls besteht kein Zweifel daran, dass nach peroraler Zufuhr von grossen Mengen Wasserswelcher Art der Mechanismus der Wasserdiurese immer auch sein mag -mannigfache Veränderungen in der Beschaffenheit des Blutes auftreten, und da deratige Veränderungen sicherlich zum Teil auf die Wasser- und Stoffmobilisationen aus der Leber, Muskulatur und anderen Depotorganen zurückzuführen sind, so kann man sich leicht vorstellen, dass bei den Mobilisationen, wie sie eben angeführt worden sind, am Bluteiweiss nicht allein quantitative Veränderung, sondern auch qualitativirgendwelche Veränderung stattfindet. Wenn dem so ist, wird es äusserst interessante Beiträge zum Einblick in die Beziehung zwischen Wasserzufuhr und Eiweissmobilisation liefern können, wenn man nach oraler Zufuhr von grossen Wassermengen, neben Bestimmungen des Hämoglobingehaltes und der Serumeiweisskonzentration die Bestimmung von k.o.D. des Blutes vornimmt. Kylin, ${ }^{18)} \mathrm{Pellegrini}{ }^{19)}$ und Meyer ${ }^{20)}$ haben diesbezügliche Untersuchungen in der Absicht ausgeführt, durch die Bestimmung von k. o. D. des Blutes das Wesen der Wasserdiurese aufzuklären.

Das Ziel vorliegenden Versuchs war aber nicht gerade hauptsächlich daraufhin gerichtet, dem Wesen der Wasserdiurese näher zu treten, sondern ich wollte mich darüber orientieren, wie sich die Eiweissmobilisation aus Depotorganen dann vollzieht, und ferner, welcher Art das hierbei mobilisierte Eiweiss ist, wenn man die perorale Zufuhr von grossen Wassermengen, also den Wasserstoss vornimmt. Von diesem Stand punkt ausgehend wurden im vorliegenden Versuch diesbezügliche Bestimmungen zuerst an gesunden Personen, danach auch an Hoch- 
druck- und Nierenkranken ausgeführt. Angesichts der bisher durch zahlreiche Untersuchungen erwiesenen Tatsache, dass bei Hochdruckund Nierenkranken die nach oraler Wasserzufuhr auftretende Verschiebung des Blutwassers, also die Veränderung in Blutbestandteilen von der Norm abweicht, erscheint es übrigens von vornherein selbstverständlich, dass bei derartigen Erkrankungen der k. o. D. des Blutes nach oraler Wasserzufuhr irgend welche Abweichungen aufweisen.

Versuchsinethode: Es standen als normales Versuchsmaterial 7 gesunde Personen zur Verfügung, davon betrafen 4 Fälle Bedienten an hiesiger Klinik und ihre Bekannten, bei übrigen 3 Fällen handelte es sich um die in hiesiger Klinik aufgenommenen Kranken mit ganz leichtgradiger Wirbelkaries, welche nach Genesung von der betreffenden Krankheit in den Versuch einweilligten. Die Hcohdruck- und Nierenkranken betrafen solche, die nach Aufnahme in hiesige Klinik genauer Untersuchung unterzogen worden sind.

Die orale Wasserzufuhr wurde nach der Methode des Volhard'schen Wasserversuchs ausgeführt, im übrigen gab man nach Wasserzufuhr Versuchspersonen die Trockendiät wie üblich und verfolgte so zugleich das Verdiinnungs- und Konzentrationsvermögen der Niere. Selbstverständlich ist es erwünscht, die Blutproben möglichst häufig in verschiedenen Zeitabständen zu entnehmen; da es aber aus manchen Gründen schwierig war, bei allen Versuchspersonen die Blutproben zu häufig zu entnehmen, wurde die Blutentnahme dreimal, unmittelbar vor Anstellung des Wasserversuchs, 2 Stunden nach der Wasserzufuhr, wo Diurese aufs stärkste gesteigert erscheint, und 24 Stunden später vorgenommen.

Die seit dem Abend vor dem Versuchstag nüchterne Versuchsperson liess man um $7 \mathrm{Uhr}$ morgens urinieren und gab $\mathrm{nm}$ morgens $8 \mathrm{Uhr}$, sofort nach Bestimmung der Harnmenge, die von $7 \mathrm{Uhr}$ ab bis $8 \mathrm{Uhr}$ morgens entleert wurde, $1,500 \mathrm{ccm}$ lauwarmes Wasser innerhalb von ca. 20 Minuten auszutrinken. Die Versuchsperson liess man von morgens $8 \mathrm{Uhr}$ ab während 4 Stunden alle halben Stunden, dann bis $8 \mathrm{Uhr}$ p. m. alle 2 Stunden urinieren, notierte jede einzelne Harnmenge und das spezifische Gewicht und sammelt die Harnmengen, die von $8 \mathrm{Uhr}$ p. m. bis zum nächsten Morgen $8 \mathrm{Uhr}$ entleert wurde. An der einzelnen Urinportion wurde die Menge und das spezifische Gewicht bestimmt. Das Körpergewicht wurde unmittelbar vor Wasserzufuhr, im direkten Anschluss daran, 4 Stunden und 24 Stunden später, im ganzen also 4 Male gemessen. Nachdem um $8 \mathrm{Uhr}$ morgens am Versuchstag Wasser gegeben worden war, wurde kein Wasser bis zum Abschluss des 24 stündigen Versuchs gegeben. Im übrigen wurde als Diät nur getrocknetes Biskuit allein dargereicht. Während der Versuchsdauer von 24 Stunden liess man auch gesunde Individuen möglichst ruhig liegen. 
Dio Blutproben wurden bei den auf der Herzhöhe gelegenen Armen der Versuchspersonen aus der V. mediana ohne Stauung mit Spritze entnommen. Der Hämoglobingehalt wurde mit dem Fle is chl-Mi e sch e r schen Hämometer, das Serumeiweiss mittels des Pulf rich schen Eintauchrefraktmeters, der k. o.D.nach Krogh und Nakaza wa ${ }^{21)}$ die Serumchlormenge nach Rus znyák bestimmt. Um neben dem Wasserversuch auch das Ausscheidungsvermögen des Farbstoffs zu untersuchen, wurden, indem man 2 Stunden nach der Wasserzufuhr $1 \mathrm{ccm}$ einer 0,6\% igen Phenolsulfonphthaleinlösung intragluteal injizierte, der Zeitpunkt, wo sich der Farbstoff auszuscheiden begann, sowie die in darauffolgenden 2 Stunden ausgeschiedene Menge mit dem Dunning'schen Kolorimeter gemessen.

\section{Versuch an normalen Meuschen.}

Die Resultate des Wasserversuchs und der Blutuntersuchung, welche an 7 gesunden Personen angestellt wurden, sind in Tab. 1 zusammengefasst.

Die Resultate des Wasserversuchs sind, von allen Seiten betrachtet, als ganz normal anzusehen. Das Maximum der Diurese trat nämlich um 2 Stunden herum nach der Wasserzufuhr auf, die in den ersten 4 Stunden ausgeschiedenen Harnmengen übertrafen in allen Fällen die zugeführte Wassermenge, die Wasserbilanz ergab sich stets negativ. Auch die Verdünnungs- und Konzentrationsfähigkeit der Niere war völlig normal erhalten, indem der Harn beim Höhepunkt der Diurese ein spezifisches Gewicht von annähernd Null zeigte und nach 12-24 Stunden hinreichend konzentriert war. Auch die Phthaleinausscheidung erfolgte ganz normal. Es lässt sich also sagen, dass bei hier untersuchten Versuchspersonen der intermediäre Wasseraustausch nach allen Richtungen hin als normal anzusehen ist. Diese Verhältnisse sind auf der vorderen Hälfte der Tabelle 1 aufgezeichnet.

Auf der Blutseite war das Hämoglobin nach 2 Stunden in 5 von 7 Fällen (Fall 3, 4, 5, 6 und 7) deutlich vermehrt, während es in übrigen 2 Fällen (Fall 1 und 2) keine nennenswerten Schwankungen aufwies. Der Hämoglobinkonzentration nach beurteilt erfuhr das Blut nach 2 Stunden tatsächlich eine Eindickung. Nach 24 Stunden wurde das Hämoglobin in solchen Fällen (Fall 1 und 2), bei denen nach 2 Stunden keine Zunahme erfolgt war, vermehrt gefunden, in denjenigen Fällen aber, bei denen sich der Hämoglobingehalt nach 2 Stunden schon als erhöht erwiesen hatte, wurden dieselben erhöhte Werte wie nach 2 Stunden oder mehr oder weniger herabgesetzte, jedenfalls gegenüber dem Anf̣angswert noch erhöhte Werte angetroffen.

21) Krogh u. Nakazawa, Biochem. Ztschr., 1927, 188, 241. 
Tabelle

Versuch an

\begin{tabular}{|c|c|c|c|c|c|c|c|}
\hline \multirow{3}{*}{ Fall } & \multirow{3}{*}{$\begin{array}{l}\text { Name, } \\
\text { Alter u. } \\
\text { Gesch- } \\
\text { lecht. }\end{array}$} & \multicolumn{5}{|c|}{ Resultate d. Wasserversuchs } & \\
\hline & & \multirow{2}{*}{$\begin{array}{l}\text { Harn- } \\
\text { menge } \\
\text { in } 24 \\
\text { Stdn. } \\
(\mathrm{ccm})\end{array}$} & \multirow{2}{*}{$\begin{array}{l}\text { Bilanz } \\
\text { in } 4 \\
\text { Stdn. } \\
(\mathrm{ccm})\end{array}$} & \multirow{2}{*}{$\begin{array}{c}\text { Min. u. } \\
\text { max. Spez. } \\
\text { Gewicht } \\
\text { d. Urins }\end{array}$} & \multicolumn{2}{|c|}{ Phthaleinprobe } & \multirow{2}{*}{$\begin{array}{l}\text { Zeit d. } \\
\text { Blutent. } \\
\text { nahme } \\
\text { (Std.) }\end{array}$} \\
\hline & & & & & $\begin{array}{c}\text { Beginn } \\
\text { (Min.) }\end{array}$ & $\begin{array}{l}\text { Innerhalb } \\
\text { 2 Stdn. } \\
\text { ausgeschie- } \\
\text { dene Menge } \\
(\%)\end{array}$ & \\
\hline 1 & $\begin{array}{l}\text { K. I. } \\
24 \\
\text { m }\end{array}$ & 2,314 & -170 & $1,000-1,030$ & 5 & 70 & $\begin{array}{l}\text { Vor } \\
\text { Nach } \\
\quad 2 \\
\quad 24\end{array}$ \\
\hline 2 & $\begin{array}{c}\text { T. A. } \\
23 \\
\text { w }\end{array}$ & 2,222 & -270 & $1,000-1,031$ & 10 & 60 & $\begin{array}{l}\text { Vor } \\
\text { Nach } \\
\quad 2 \\
\Rightarrow \quad 24\end{array}$ \\
\hline 3 & $\begin{array}{c}\text { E. K. } \\
29 \\
\text { m }\end{array}$ & 2,480 & -560 & $1,000-1,030$ & 5 & 70 & $\begin{array}{ll}\text { Vor } & \\
\text { Nach } & 2 \\
, \quad 24\end{array}$ \\
\hline 4 & $\begin{array}{c}\text { K. K. } \\
34 \\
\mathrm{~m}\end{array}$ & 2,440 & -460 & $1,000-1,031$ & 5 & 65 & $\begin{array}{l}\text { Vor } \\
\text { Nach } 2 \\
\Rightarrow \quad 24\end{array}$ \\
\hline 5 & $\begin{array}{l}\text { S. M. } \\
20 \\
\text { m }\end{array}$ & 2,630 & -660 & $1,000-1,032$ & 5 & 80 & $\begin{array}{ll}\text { Vor } & \\
\text { Nach } & 2 \\
& 24\end{array}$ \\
\hline 6 & $\begin{array}{c}\mathrm{K} . \mathrm{T} . \\
20 \\
\mathrm{~m}\end{array}$ & 2,400 & -460 & $1,000-1,032$ & 5 & 70 & $\begin{array}{l}\text { Vor } \\
\text { Nach } \\
\quad 2 \\
\Rightarrow \quad 24\end{array}$ \\
\hline 7 & $\begin{array}{c}\text { K. I. } \\
29 \\
\text { m }\end{array}$ & 2,650 & -780 & $1,000-1,031$ & 5 & 65 & $\begin{array}{ll}\text { Vor } & \\
\text { Nach } & 2 \\
& 24\end{array}$ \\
\hline
\end{tabular}

Serumeiweiss zeigte in Fällen, wo die Hämoglobinzunahme stattfand, auch gewisse Vermehrung, die aber geringer als die des Hämoglobins war, indem nach 2 Stunden sich die prozentische Zunahme des Hämoglobins auf 2,6-6,4, die des Eiweisses auf 1,3-5,4 beläuft. Nach 24 Stunden traten erhebliche Schwankungen an beiden Werten, allerdings übertraf auch hier die Hämoglobinzunahme im allgemeinen die Eiweisszunahme, indem das erstere eine prozentische Zunahme von 1,0-8,2 aufwies, während die prozentische Zunahme des letzteren 0,55,7 betrug. Als ein Beispiel hierfür ist die prozentische $A b$ - und Zunahme der beiden Werte in Versuch 3 neben dem Ergebnis des Wasserversuchs kurvenmässig dargestellt (Fig. 1). 
1.

Gesunden.

\begin{tabular}{|c|c|c|c|c|c|c|c|c|}
\hline \multicolumn{9}{|c|}{ Blutuntersuchung. } \\
\hline \multicolumn{2}{|c|}{ Hämoglobin } & \multicolumn{2}{|c|}{ Serumeiweiss } & \multicolumn{2}{|c|}{ K. o. D. } & \multicolumn{2}{|c|}{ Druck pro $q_{0}$} & \multirow{2}{*}{$\begin{array}{c}\text { Serum-NaCl } \\
(\mathrm{g} / \mathrm{dl})\end{array}$} \\
\hline $\mathrm{g} / \mathrm{dl}$ & $\underset{\%}{\text { Diff. in }}$ & $\therefore$ & $\begin{array}{c}\text { Diff. in } \\
\%\end{array}$ & $\mathrm{mmH}_{2} \mathrm{O}$ & Diff. in & $\mathrm{mmH}_{2} \mathrm{O}$ & $\begin{array}{c}\text { Diff. in } \\
\%\end{array}$ & \\
\hline $\begin{array}{l}16,92 \\
16,92 \\
17,92\end{array}$ & $\begin{array}{l}+0 \\
+5, y\end{array}$ & $\begin{array}{l}7,72 \\
8,08 \\
8,04\end{array}$ & $\begin{array}{l}+4,7 \\
+4,1\end{array}$ & $\begin{array}{l}361 \\
370 \\
380\end{array}$ & $\begin{array}{l}+2,5 \\
+5,3\end{array}$ & $\begin{array}{l}46,8 \\
45,8 \\
47,3\end{array}$ & $\begin{array}{l}-2,1 \\
+1,1\end{array}$ & $\begin{array}{l}0,540 \\
0,535 \\
0,540\end{array}$ \\
\hline $\begin{array}{l}14,58 \\
14,49 \\
14,80\end{array}$ & $\begin{array}{l}-0,6 \\
+1,5\end{array}$ & $\begin{array}{l}8,33 \\
8,17 \\
8,56\end{array}$ & $\begin{array}{l}-1,9 \\
+2,8\end{array}$ & $\begin{array}{l}377 \\
368 \\
403\end{array}$ & $\begin{array}{l}-2,4 \\
+6,9\end{array}$ & $\begin{array}{l}45,2 \\
45,0 \\
47,1\end{array}$ & $\begin{array}{l}-0,4 \\
+4,2\end{array}$ & $\begin{array}{l}0,615 \\
0,605 \\
0,615\end{array}$ \\
\hline $\begin{array}{l}13,94 \\
14,80 \\
15,08\end{array}$ & $\begin{array}{l}+6,2 \\
+8,2\end{array}$ & $\begin{array}{l}8,28 \\
8,56 \\
8,71\end{array}$ & $\begin{array}{l}+3,4 \\
+5,2\end{array}$ & $\begin{array}{l}366 \\
372 \\
398\end{array}$ & $\begin{array}{l}+1,6 \\
+8,7\end{array}$ & $\begin{array}{l}44,2 \\
43,4 \\
45,7\end{array}$ & $\begin{array}{l}-1,8 \\
+3,4\end{array}$ & $\begin{array}{l}0,605 \\
0,590 \\
0,590\end{array}$ \\
\hline $\begin{array}{l}13,08 \\
13,42 \\
13,36\end{array}$ & $\begin{array}{l}+2,6 \\
+2,1\end{array}$ & $\begin{array}{l}7,13 \\
7,31 \\
7,27\end{array}$ & $\begin{array}{l}+2,5 \\
+2,0\end{array}$ & $\begin{array}{l}303 \\
307 \\
319\end{array}$ & $\begin{array}{l}+1,3 \\
+5,3\end{array}$ & $\begin{array}{l}42,5 \\
42^{6} 0 \\
43,9\end{array}$ & $\begin{array}{l}-1,2 \\
+3,3\end{array}$ & $\begin{array}{l}0,625 \\
0,615 \\
0,625\end{array}$ \\
\hline $\begin{array}{l}13,64 \\
14,52 \\
14,52\end{array}$ & $\begin{array}{l}+6,4 \\
+6,4\end{array}$ & $\begin{array}{l}7,55 \\
7,72 \\
7,78\end{array}$ & $\begin{array}{l}+2,2 \\
+3,0\end{array}$ & $\begin{array}{l}341 \\
340 \\
352\end{array}$ & $\begin{array}{l}-0,3 \\
+3,2\end{array}$ & $\begin{array}{l}45,2 \\
44,0 \\
45,2\end{array}$ & $\begin{array}{l}-2,6 \\
\pm 0\end{array}$ & $\begin{array}{l}0,577 \\
0,570 \\
0,570\end{array}$ \\
\hline $\begin{array}{l}14,24 \\
15,08 \\
14,52\end{array}$ & $\begin{array}{l}+5,9 \\
+2,0\end{array}$ & $\begin{array}{l}8,00 \\
8,43 \\
8,15\end{array}$ & $\begin{array}{l}+5,4 \\
+1,9\end{array}$ & $\begin{array}{l}372 \\
390 \\
383\end{array}$ & $\begin{array}{l}+4,8 \\
+2,9\end{array}$ & $\begin{array}{l}46,5 \\
46,3 \\
47,0\end{array}$ & $\begin{array}{l}-0,4 \\
+1,1\end{array}$ & $\begin{array}{l}0,600 \\
0,605 \\
0,600\end{array}$ \\
\hline $\begin{array}{l}14,24 \\
14,80 \\
14,38\end{array}$ & $\begin{array}{l}+3,9 \\
+1,0\end{array}$ & $\begin{array}{l}8,49 \\
8,60 \\
8,54\end{array}$ & $\begin{array}{l}+1,3 \\
+0,6\end{array}$ & $\begin{array}{l}414 \\
402 \\
420\end{array}$ & $\begin{array}{l}-2,9 \\
+1,4\end{array}$ & $\begin{array}{l}48,7 \\
46,7 \\
49,1\end{array}$ & $\begin{array}{l}-4,1 \\
+0,8\end{array}$ & $\begin{array}{l}0,565 \\
0,555 \\
0,565\end{array}$ \\
\hline
\end{tabular}

Von 2 Versuchen, wo in 2 stündigem Ablauf nach Wasserzufuhr keine Hämoglobinzunahme aufgetreten war, wurde bei einem (Versuch 1) das Eiweiss, trotzdem der Hämoglobingehalt keine Zunahme erfuhr, erheblich vermehrt gefunden, diese prozentische Zunahme des Eiweisses betrug 4,7(Fig, 2). In einem andern Versuch (Versuch 2) hingegen nahm das Eiweiss, im Verein mit geringer Hämoglobinabnahme, ab.

Aus dem Verhalten des Eiweisses und Hämoglobins im 2 stündigen sowie 24 stündigen Ablauf geht klar hervor, dass in 7 Versuchen im grossen und ganzen die Konzentrationszunahme des Hämoglobins immer mit der des Eiweisses verbunden war, wobei aber die Zunahme des ersteren derselben des letzteren ïberlegen war, und aus dem Umstand, 
Fig. 1. Veränderungen des Serumeiweisses und dessen kolloid-osmotischen Drucks durch orale Wasserzufuhr (Wasserversuch nach Volhard) bei Gesunden (Fall 3).

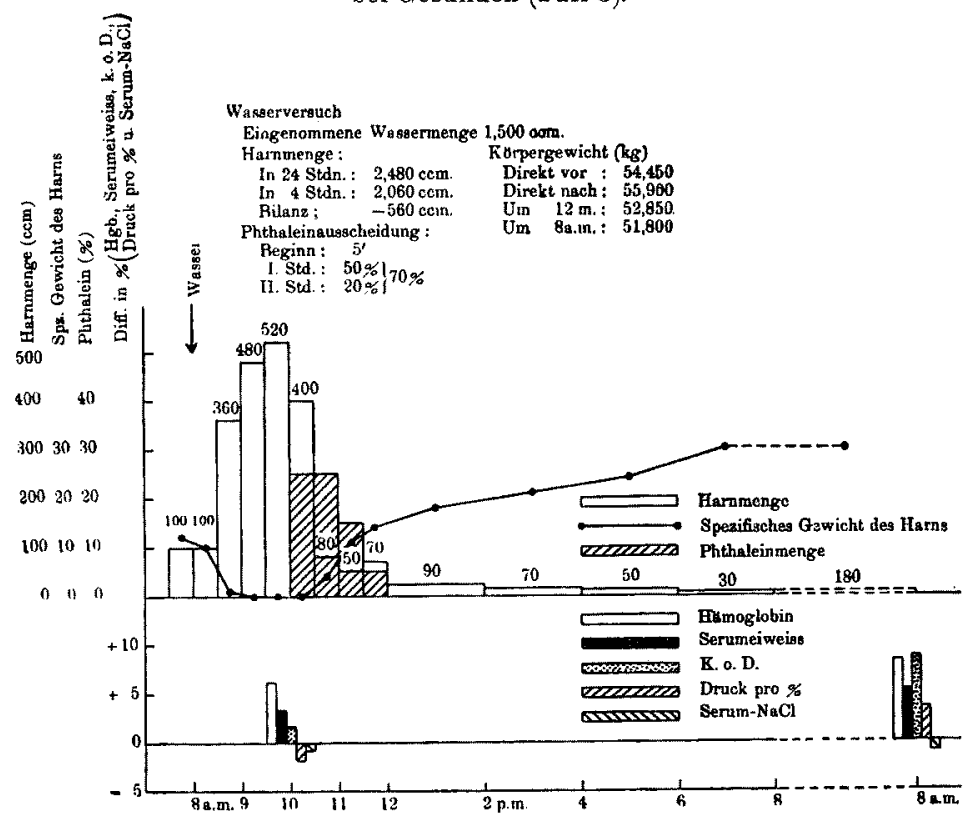

dass es Fälle gab, wo das Eiweiss beim Ausbleiben der Hämoglobinzunahme beträchtliche Zunahme aufwies, kann geschlossen werden, dass diese quantitative Veränderung des Eiweisses nicht etwa eine sekundäre, auf der Veränderung der Blutkonzentration beruhende Erscheinung ist, sondern dass durch den Wasserstoss das Bluteiweiss für sich seine eigene Veränderung dokumentiert.

Es drängte sich dann die Frage auf, in welcher Weise das Bluteiweiss qualitativ beeinflusst wurde. Wenn man dies von der Seite des k. o. D. betrachtet, so erkennt man, dass von 6 Versuchen, in denen nach 2 Stunden die Eiweisszunahme auftrat (das Eiweiss nahm nur in Versuch $2 \mathrm{ab}$, in allen übrigen Versuchen zu) in 4 der k. o. D. in 2 stündigem Ablauf erhöht war, während er in übrigen 2 Versuchen und in Versuch 2, wo die Eiweisszunahme vermisst wurde, eine Herabsetzung erfuhr. In 2 stündigem Ablauf nach der Wasserzufuhr nahm der k. o. D. also beinahe in der Hälfte der Versuche ab und in der anderen Hälfte zu, so dass man, wie $\mathrm{Meyer}{ }^{20)}$ hervorgehoben hat, weder von der Abnahme noch von derZunahme sprechen kann. Da aber die prozentischeZunahme des k. o. D. stets ohne Ausnahme geringer als die des Eiweisses 
Fig. 2. Veränderungen des Serumeiweisses und dessen kolloid-osmotischen Drucks durch orale Wasserzufuhr (Wasserversuch nach Volhard) bei Gesunden (Fall 1).

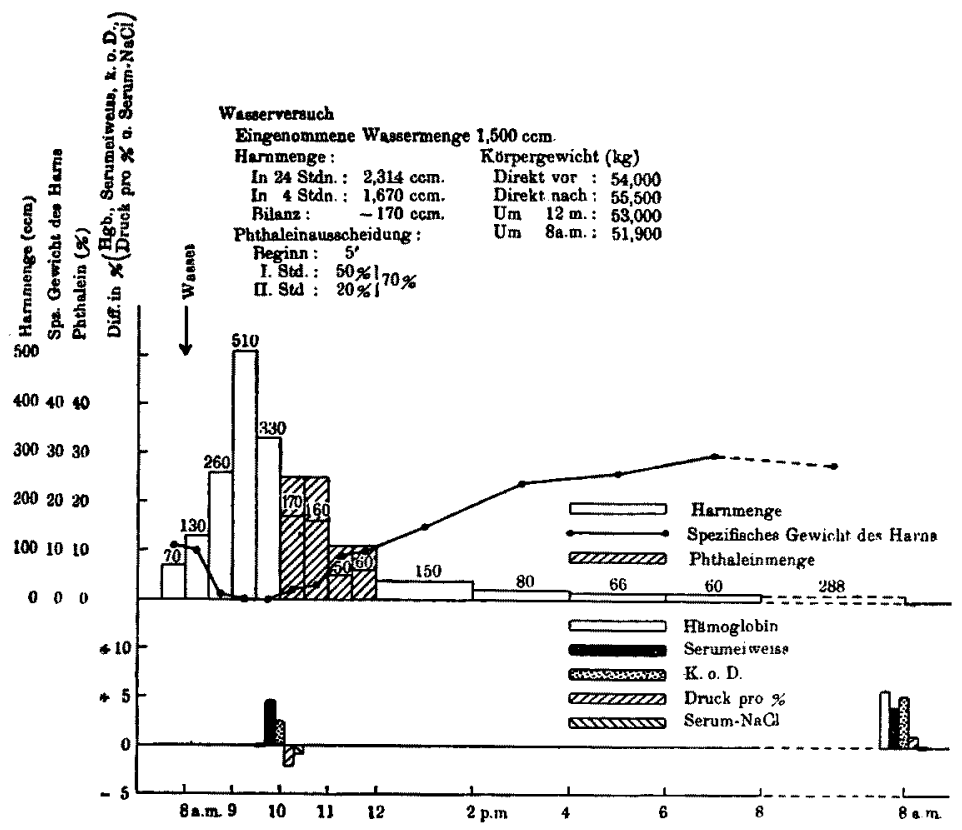

war, wies derDruck pro $\%$ in allen 7 Versuchen eine mehr oder weniger Herabsetzung auf. Es deutet dies offenbar darauf hin, dass es sich bei hier stattfindender qualitativer Veränderung des Eiweisses ausnahmslos um eine absolute oder relative Zunahme der weniger osmoaktiven Eiweissteilchen von grösseren Molekularaggregaten handelt.

In 24 stündigem Ablauf nach der Wasserzufuhr erfuhr der k. o. D. auch in Fällen, wo in 2 stündigem Ablauf eine Abnahme angetroffen wurde, eine beträchtliche Zunahme; zumal in Fällen, wo in 2 stündigem Ablauf bereits eine Zunahme des k. o. D. ermittelt wurde, erhöhte sich der k. o. D. meistens noch mehr. Dementsprechend zeigte der Druck pro \% in den meisten Fällen höhere Werte als anfänglich.

Der Serum-NaCl-Spiegel erfuhr auch eine mehr oder weniger Abnahme, welche jedoch sehr geringgradig war.

Es sei nun im folgenden an Hand der oben erhaltenen Daten darüber besprochen, wie sich in 2 stündigem und 24 stündigem Ablauf nach 
dem Wasserstoss die quantitative und qualitative Veränderung des Bluteiweisses einstellt. Bevor ich auf das Hauptthema eingehe, möchte ich mich dem Verhalten der Blutkonzentration nach oraler Wasserzufuhr zuwenden. Nach den Untersuchungen von $\mathrm{Marx}^{14)}$ und $\mathrm{A}$ b e ${ }^{17)}$ in denen beide Forscher den Wasserversuch nach Volhard am Menschen anstellten und die nach Wasserzufuhr auftretenden Veränderungen in der Blutkonzentration eingehend studierten, soll unmittelbar nach der Wasserzufuhr eine initielle Hydrämie aufgetreten sein, die aber bald darauf verschwand, schon 2 Stunden nach der Wasserzufuhr trat die Phase der primären Bluteindickung ein, worauf eine sekundäre Hydrämie folgte, die aber, weil man nur die Trockenkost, kein Wasser gab, allmählich in die terminale Bluteindickung überging. Auch in meinem Versuch fiel in die zweite Stunde nach der Wasserzufuhr gerade die Phase der primären Bluteindickung, wobei dieZunahme der Hämoglobinkonzentration in den meisten Fällen auftrat. Nach 24 Stunden wurde die Phase der terminalen Bluteindickung mit gleichzeitiger Konzentrationszunahme des Hämoglobins angetroffen.

Was nun die Veränderungen des Bluteiweisses, die 2 Stunden nach der Wasserzufuhr auftraten, anbelangt, erhöhte sich die Eiweisskonzentration, wie bereits angeführt, beim Auftreten der Hämoglobinzunahme in den meisten Fällen; der Grad dieser Erhöhung stand jedoch demselben der Hämoglobinzunahme nach. Mitunter gab es Fälle, in denen das Eiweiss beim Ausbleiben der Hämoglobinkonzentrationserhöhung doch zunahm. Derartige Eiweisszunahme dürfte zwar zum Teil durch die Bluteindickung bedingt sein, man muss aber zugleich ausserdem noch 2 Faktoren in Betracht ziehen. Erstens ist es das Eindringen der Eiweissteilchen vom Blute ins Gewebe; als der zweite Faktor ist wohl aufzufassen der Einstrom der Bluteiweisskörper aus Eiweissdepotorganen, wie Leber und Muskulatur, in die Blutbahn, also der mit der Wasserverschiebung im Organismus einhergehende Austausch der Eiweissteilchen. Es ist von Korányi, ${ }^{22)} \mathrm{Marx}^{23)}$ und Baer ${ }^{24)}$ erkannt worden, dass in der Phase der primären Bluteindickung dargereichtes Wasser in die Leber oder die Muskeln übergeht, wodurch es zur Volumzunahme der betreffenden Organe kommt. Und der Umstand, dass in eben erwähnter Phase das Eiweiss zwar eine Zunahme aufweist, die aber derselben des Hämoglobins an Grad nach-

22) Korányi, Vorlesungen über funktionelle Pathologie und Therapie der Nierenkrankheiten, Berlin 1929, 92.

23) Ma rx, Wasserhaushalt des Menschen, Berlin 1935, 52.

24) B a er, Areh. f. exp. Path. u. Pharmak., 1927, 119, 102. 
steht, ist darauf zurückzuführen, dass ein Teil des Bluteiweisses samt dem Wasser ins Gewebe hineinströmt. Darüber, dass bei peroraler Zufuhr von grossen Wassermengen sich eine derartige Eiweissmobilisation zwischen Blut und Geweben vollzieht, ist bereits von Gollow itzer-Meier u. Rabl' berichtet worden. Nach alledem ist anzunehmen, dass, weil das hierbei ins Gewebe eingedrungene Eiweiss aus relativ osmoaktiven feindispersen Eiweissteilchen zusammengesetzt ist und deshalb im Blut relativ grobdisperse Eiweissteilchen zurückbleiben, der k. o. D. eine gegenüber der Eiweisszunahme geringere Erhöhung aufweist, was eine Erniedrigung des Drucks pro \% zur Folge haben dürfte. Es gibt aber denjenigen Fall wie Versuch 1, wo sich beim Konstantbleiben der Hämoglobinkonzentration eine Eiweisszunahme zeigte. Diese Eiweisszunahme kann durch die infolge des Anreizes der Wasserzufuhr eintretende Mobilisation des Eiweisses aus Depotorganen bedingtsein, oder aber man kann auch daran denken, dass, weil bei diesem Fall, wie aus beigefügter Figur ersichtlich ist, sich die Wasserausscheidung sehr geschwind vollzieht, das Blut schon an diesem Zeitpunkt auf dem Wege zu sekundärer Hydrämie befindet, wobei Eiweisszunahme noch bestehen bleibt, während das Hämoglobin schon auf den Ursprungswert zurückgekommen ist. Immerhin, aus jener, gegenüber der Eiweisszunahme weitaus geringeren Erhöhung des k. o. D. sowie aus der Erniedrigung des Drucks pro \% muss zwangsläufig geschlossen werden, dass das hierbei mobilisierte Eiweiss aus den weniger osmoaktiven Eiweissteilchen von grösseren Molekularaggregaten besteht.

Alles in allen dürfte die Annahme wohl gerechtfertigt sein, dass die Erniedrigung des Drucks pro \% dadurch zustande kommt, dass in 2 stündigem Ablauf nach peroraler Wasserzufuhr das Blut seinen Bestand an kleinen Eiweissteilchen samt dem Wasser an die Gewebe abgibt oder grössere Eiweissteilchen aus den Depots aufnimmt. Sehr wahrscheinlich dürften in 2stündigem Zeitablauf im Blut zweierlei Austauschvorgänge, wie oben geschildert, nämlich das Hineindringen kleiner Eiweissteilchen ins Gewebe sowie der Einstrom grösserer Eiweiss teilchen ins Blut mit im Spiel sein, und je nach der Intensität dieser Vorgänge dürften wohl die Zunahme des Bluteiweisses, die Erhöhung des k. o. D., die Erniedrigung des Drucks pro \% usw. in verschieden starken Graden zum Vorschein kommen.

Auch von anderem Gesichtspunkt aus kann man den Beweis dafür führen, dass die Veränderung des Serumeiweisses nicht durch eine ein- 
fache Bluteindickung bedingt ist. Nach Untersuchungen von May r s, ${ }^{25)}$ Verney, ${ }^{26)}$ Krogh u. Nakazawa ${ }^{21)}$ erhöht sich der Druck pro \% schon bei bloss mechanischer Eindickung der Blutkolloide wenn auch in geringem Masse. In vorliegendem Versuch indessen sinkt derDruck pro \% hingegen trotz der gemeinsamen Zunahmen des Eiweisses und Hämoglobins stets ab. Aus dieser Tatsache geht allerdings hervor, dass es sich hier nicht um eine einfache Bluteindickung handelt. $\mathrm{Da}$ aber in 24 stündigem Ablauf die Bluteindickung immer mit Erhöhungen des k. o. D. und Drucks pro \% einhergeht, dürfte die Eiweissveränderung in dieser Zeitspanne wahrscheinlich in der Hauptsache als eine sekundäre, von der Erhöhung der Blutkonzentration begleitete Erscheinung aufzufassen sein.

Kurzum, es lässt sich also sagen, dass die Veränderungen des Bluteiweisses, die 2 Stumden nach peroraler Wasserzufuhr bei gesunden Menschen auftreten, recht kompliziert und eingreifend sind, während die Veränderungen nach 24 Stunden einfach als Folge einer Bluteindickung anzusehen sind.

\section{Versuch an essentiellen Hochdruckkranken.}

Resultate des an 12 Fällen von essentieller Hypertonie ausgeführten Wasserversuchs mit der Blutuntersuchung sind in Tab. 2 zusammengestellt.

Nach dem Ergebnisse des Wasserversuchs zeigen bei diesen Kranken das Verdünnungs- und Konzentrationsvermögen sowie die Phthaleinausscheidung gegenüber Gesunden keine grossen Unterschiede. Die Wasserausscheidung war im wesentlichen nicht als besonders gestört zu betrachten, wohl aber gegenüber im vorherigen Kapitel geschilderten gesunden Individuen meistens mehr oder weniger verzögert. Den in den ersten 4 Stunden ausgeschiedenen Harnmengen nach beurteilt, wurde nämlich bei 4 von 12 Fällen aufgenommenes Wasser nicht völlig ausgeschieden, es bestand hier eine positive Wasserbilanz; bei übrigen 8 Fällen wurde zwar eine negative Wasserbilanz gefunden, welche aber, wie aus Tabb. 1 und 2 anschaulich ist, meistens zwischen 100 bis $300 \mathrm{ccm}$ schwankte, die also im allgemeinen kleiner als bei Gesunden war. Es lässt sich allerdings sagen, dass die Wasserausscheidung im grossen ganzen ein wenig verzögert war.

25) Ma y r s, Quart. Journ. Med, 1926, 19, 273.

26) Verney, Journ. Physiol., 1926, 61, 319. 
In jüngster Zeit äusserte sich Ma rx ${ }^{27)}$ dass bei essentieller $\mathrm{Hy}$ pertonie die nach peroraler Wasserzufuhr eintretende initielle $\mathrm{Hy}$ drämie und die nachfolgende primäre Bluteindickung in gleichem Verhältnis wie bei Gesunden stünden. Nach meinen Untersuchungen aber waren sie infolge mässig verzögerter Wasserausscheidung auch zeitlich gewissermassen verschoben.

Was die 2 Stunden nach der Wasserzufuhr aufgetretenen Veränderung am Blut anbelangt, erfuhr die Hämoglobinkonzentration im Gegensatz zu Gesunden in keinem Fall die Zunahme, indem sie in 7 von 12 Fällen abnahm und in 5 Fällen annähernd gleiche Werte wie Ursprungswerte aufwies. Aus dem Verhalten der Hämoglobinkonzentration und Wasserausscheidung schliessend, dürfte das Blut in denjenigen Fällen, wo die Hämoglobinkonzentration herabgesetzt war, in diesem Zeitpunkt noch nicht von initieller Hydrämie erholt sein. Bei solchen Fällen, wo die Hämoglobinkonzentration den annähernden Vorwert aufwies, dürfte sich das Blut auf dem Übergang von initieller Hydrämie zu primärer Eindickung befinden.

In dieserZeitspanne nahm das Eiweiss ohne Ausnahme ab. Wenn man bei 7 Fällen, in denen die Abnahme der Hämoglobinkonzentration auftrat, dieselbe mit der Eiweissabnahme vergleicht, so erkennt man, dass bei 3 Fällen (Fall 1, 3 und 4) die prozentische Abnahme des Eiweisses geringer als die des Hämoglobins, während bei 2 Fällen (Fall 6 und 12) die Abnahme beider Werte fast gleich war, und bei übrigen 2 Fällen (Fall 7 und 8) hingegen die erstere grösser als die letztere war. Bei 5 Fällen, wo eine bemerkbare Hämoglobinabnahme vermisst wurde, trat ohne Ausnahme eine Abnahme des Eiweisses auf. Auch in diesem Fall ging also die Eiweissabnahme dem Verhalten des Hämoglobins nicht parallel. Hieraus geht offensichtlich hervor, dass hier die quantitative Veränderung des Eiweisses, in Analogie zu Gesunden, durchaus nicht eine sekundäre, mit der Konzentrationsänderung des Blutes einhergehende Begleiterscheinung war.

Im folgenden werden die qualitativen Veränderungen des Eiweisses von derSeite des k. o. D. betrachtet und die diese Veränderungen bedingenden Momente unter Einteilung in mehrere Formen auseinandergesetzt.

1. Fälle mit Abnahmeder Hämoglobinkonzentration, diestärker als die des Serumeiweissesist (Fall 1, 3 und 4): Die prozentische $\mathrm{Ab}$ - und Zunahme der betreffenden Grössen beim Fall

27) Ma x x, Dtsch. Arch. f. klin. Med., 1926, 152, 354. 
Tabelle

Versuch an

\begin{tabular}{|c|c|c|c|c|c|c|c|}
\hline \multirow[b]{3}{*}{ Fall } & \multirow{3}{*}{$\begin{array}{l}\text { Name, } \\
\text { Alter u. } \\
\text { Gesch- } \\
\text { lecht }\end{array}$} & \multicolumn{5}{|c|}{ Resultate $d$. Wasserversuchs } & \\
\hline & & \multirow{2}{*}{$\begin{array}{l}\text { Harn- } \\
\text { menge } \\
\text { in } 24 \\
\text { Stdn. } \\
\text { (ccm) }\end{array}$} & \multirow{2}{*}{$\begin{array}{l}\text { Bilanz } \\
\text { in } 4 \\
\text { Stdn. } \\
\text { (ccm) }\end{array}$} & \multirow{2}{*}{$\begin{array}{l}\text { Min. } u . \\
\text { max. Sp. } \\
\text { Gewicht d. } \\
\text { Urins }\end{array}$} & \multicolumn{2}{|c|}{ Phthaleinprobe } & \multirow{2}{*}{$\begin{array}{l}\text { Zeit d. } \\
\text { Blutent- } \\
\text { nahme } \\
\text { (Std.) }\end{array}$} \\
\hline & & & & & $\begin{array}{c}\text { Beginn } \\
\text { (Min.) }\end{array}$ & $\begin{array}{c}\text { Innerhalb } \\
2 \text { Stdn. } \\
\text { ausgeschie- } \\
\text { dene Menge } \\
(\%)\end{array}$ & \\
\hline 1 & $\begin{array}{c}\text { T. S. } \\
\mathbf{5 9} \\
\text { w }\end{array}$ & $\mathrm{I}, 990$ & -109 & $1,000-1,030$ & 10 & 70 & $\begin{array}{ll}\text { Vor } & \\
\text { Nach } & 2 \\
& 24 \\
\end{array}$ \\
\hline 2 & $\begin{array}{c}\text { S. T. } \\
48 \\
\text { m }\end{array}$ & 2,218 & -348 & $1,000-1,028$ & 5 & 65 & $\begin{array}{ll}\text { Vor } & \\
\text { Nach } & 2 \\
& 24 \\
\end{array}$ \\
\hline 3 & $\begin{array}{c}\text { T. M. } \\
50 \\
\mathrm{~m}\end{array}$ & 2,146 & -190 & $1,001-1,037$ & 10 & 60 & $\begin{array}{ll}\text { Vor } & \\
\text { Nach } & 2 \\
, & 24\end{array}$ \\
\hline 4 & $\begin{array}{c}\text { M. I. } \\
61 \\
\text { In }\end{array}$ & 1,649 & +280 & $1,000-1,028$ & 10 & 50 & $\begin{array}{ll}\text { Vor } & \\
\text { Nach } & 2 \\
& 24\end{array}$ \\
\hline 5 & $\begin{array}{c}\text { M. S. } \\
57 \\
\text { w }\end{array}$ & 1,519 & +165 & $1,000-1,032$ & 10 & 90 & $\begin{array}{l}\text { Vor } \\
\text { Nach } \\
\quad 2 \\
\Rightarrow \quad 24\end{array}$ \\
\hline 6 & $\begin{array}{c}Y . Y \\
54 \\
W\end{array}$ & 2,137 & -325 & $1,002-1,025$ & 10 & 70 & $\begin{array}{ll}\text { Vor } & \\
\text { Nach } & 2 \\
& 24\end{array}$ \\
\hline 7 & $\begin{array}{c}\text { B. N. } \\
53 \\
\mathrm{~m}\end{array}$ & 1,993 & -66 & $1,000-1,027$ & 5 & 80 & $\begin{array}{ll}\text { Vor } & \\
\text { Nach } & 2 \\
& 24\end{array}$ \\
\hline 8 & $\begin{array}{c}\text { M. Y. } \\
58 \\
\mathrm{~m}\end{array}$ & 2,248 & +168 & $1,001-1,030$ & 5 & 65 & $\begin{array}{l}\text { Vor } \\
\text { Nach } \\
2, \quad 24\end{array}$ \\
\hline 9 & $\begin{array}{c}\text { M. G. } \\
65 \\
\text { w }\end{array}$ & 1,955 & -55 & $1,000-1,030$ & 10 & 60 & $\begin{array}{ll}\text { Vor } & \\
\text { Nach } & 2 \\
, \quad 24\end{array}$ \\
\hline 10 & $\begin{array}{c}\text { H. S. } \\
59 \\
\mathrm{~m}\end{array}$ & 2,200 & -60 & $1,000-1,028$ & 10 & 70 & $\begin{array}{ll}\text { Vor } & \\
\text { Nach } & 2 \\
n & 24\end{array}$ \\
\hline 11 & $\begin{array}{c}\text { Y. W. } \\
\mathbf{5 6} \\
\mathrm{m}\end{array}$ & 2,142 & -212 & $1,002-1,025$ & 10 & 60 & $\begin{array}{l}\text { Vor } \\
\text { Nach } \\
y^{\circ} \quad 24\end{array}$ \\
\hline 12 & $\begin{array}{l}\text { M. M. } \\
37 \\
w\end{array}$ & 1,713 & +188 & $1,000-1,025$ & 10 & 50 & $\begin{array}{l}\text { Vor } \\
\text { Nach } \\
\Rightarrow \quad 24\end{array}$ \\
\hline
\end{tabular}

1 ist als ein Beispiel kurvenmässig in Fig. 3 dargestellt. Bei dieser Form befindet sich das Blut 2 Stunden nach Flüssigkeitseinnahme in einem Zustand der initiellen Hydrämie. Da nach Untersuchungen von $\mathrm{O} \mathrm{k} \mathrm{a}^{28)}$ soll hierbei die zirkulierende Blutmenge, trotzdem das zugefü-

28) Ok a, Tohoku Journ. Exp. Med. 1938, 32, 382. 
2.

essentieller Hypertonie.

\begin{tabular}{|c|c|c|c|c|c|c|c|c|c|}
\hline \multicolumn{9}{|c|}{ Blutuntersuchung } & \multirow{3}{*}{$\begin{array}{l}\text { Blut- } \\
\text { druck } \\
\text { (mmHg) }\end{array}$} \\
\hline \multicolumn{2}{|c|}{ Hämoglobin } & \multicolumn{2}{|c|}{ Serumeiweiss } & \multicolumn{2}{|c|}{ K. o. D. } & \multicolumn{2}{|c|}{ Druck pro $\%$} & \multirow{2}{*}{$\begin{array}{c}\text { Serum- } \\
\text { NaCl } \\
(\mathrm{g} / \mathrm{dl})\end{array}$} & \\
\hline $\mathrm{g} / \mathrm{dl}$ & $\begin{array}{c}\text { Diff. in } \\
\%\end{array}$ & $\%$ & $\begin{array}{c}\text { Diff. in } \\
\%\end{array}$ & $\mathrm{mmH}_{2} \mathrm{O}$ & $\begin{array}{c}\text { Diff. in } \\
\%\end{array}$ & $\mathrm{mmH}_{2} \mathrm{O}$ & $\underset{\%}{\text { Diff. in }}$ & & \\
\hline $\begin{array}{l}14,80 \\
14,23 \\
15,36 \\
\end{array}$ & $\begin{array}{r}-3,8 \\
+3,8 \\
\end{array}$ & $\begin{array}{l}8,94 \\
8,73 \\
9,01 \\
\end{array}$ & $\begin{array}{r}-2,3 \\
+0,8 \\
\end{array}$ & $\begin{array}{l}381 \\
366 \\
392 \\
\end{array}$ & $\begin{array}{r}-3,9 \\
+2,9 \\
\end{array}$ & $\begin{array}{l}42,6 \\
41,9 \\
43,5 \\
\end{array}$ & $\begin{array}{r}-1,6 \\
+2,1 \\
\end{array}$ & $\begin{array}{l}0,560 \\
0,555 \\
0,565\end{array}$ & $\begin{array}{l}212 \\
195\end{array}$ \\
\hline \begin{tabular}{|l|}
14,52 \\
14,52 \\
14,83
\end{tabular} & $\begin{array}{l} \pm 0 \\
+2,1 \\
\end{array}$ & $\begin{array}{l}8,47 \\
8,43 \\
8,71 \\
\end{array}$ & $\begin{array}{r}-0,5 \\
+2,8 \\
\end{array}$ & $\begin{array}{l}399 \\
385 \\
415 \\
\end{array}$ & $\begin{array}{r}-3,5 \\
+4,0 \\
\end{array}$ & $\begin{array}{l}47,1 \\
45,7 \\
47,6\end{array}$ & $\begin{array}{l}-3,0 \\
+1,1\end{array}$ & $\begin{array}{l}0,560 \\
0,530 \\
0,560\end{array}$ & 200 \\
\hline $\begin{array}{l}14,52 \\
13,94 \\
14,52\end{array}$ & $\begin{array}{l}-4,0 \\
\pm 0\end{array}$ & $\begin{array}{l}7,70 \\
7,50 \\
8,06\end{array}$ & $\begin{array}{l}-2,6 \\
+4,7\end{array}$ & $\begin{array}{l}338 \\
324 \\
361\end{array}$ & $\begin{array}{l}-4,1 \\
+6,8\end{array}$ & $\begin{array}{l}43,7 \\
43,2 \\
44,8\end{array}$ & $\begin{array}{l}-1,1 \\
+2,5\end{array}$ & $\begin{array}{l}0,620 \\
0,615 \\
0,615\end{array}$ & 155 \\
\hline $\begin{array}{l}14,80 \\
13,94 \\
14,52\end{array}$ & $\begin{array}{l}-5,8 \\
+1,9\end{array}$ & $\begin{array}{l}7,48 \\
7,16 \\
7,48\end{array}$ & $\begin{array}{l}-4,3 \\
\pm 0\end{array}$ & $\begin{array}{l}333 \\
321 \\
334\end{array}$ & $\begin{array}{l}-3,6 \\
+0,3\end{array}$ & $\begin{array}{l}44,5 \\
44,8 \\
44,6\end{array}$ & $\begin{array}{l}+0,7 \\
+0,2\end{array}$ & $\begin{array}{l}0,560 \\
0,540 \\
0,565\end{array}$ & 150 \\
\hline \begin{tabular}{l|}
14,72 \\
14,52 \\
15,02
\end{tabular} & $\begin{array}{l}-1,3 \\
+2,0\end{array}$ & $\begin{array}{l}9,03 \\
8,56 \\
9,16\end{array}$ & $\begin{array}{l}-5,2 \\
+1,4\end{array}$ & $\begin{array}{l}396 \\
373 \\
414\end{array}$ & $\begin{array}{l}-5,8 \\
+4,5\end{array}$ & $\begin{array}{l}43,8 \\
43,6 \\
45,2\end{array}$ & $\begin{array}{l}-0,4 \\
+3,2\end{array}$ & $\begin{array}{l}0,580 \\
0,580 \\
0,585\end{array}$ & 150 \\
\hline $\begin{array}{l}15,64 \\
14,80 \\
15,64\end{array}$ & $\begin{array}{l}-5,4 \\
\pm 0\end{array}$ & $\begin{array}{l}8,49 \\
8,00 \\
8,66\end{array}$ & $\begin{array}{l}-5,8 \\
+2,0\end{array}$ & $\begin{array}{l}391 \\
365 \\
398\end{array}$ & $\begin{array}{l}-6,6 \\
+1,8\end{array}$ & $\begin{array}{l}46,0 \\
45,6 \\
45,9\end{array}$ & $\begin{array}{l}-0,9 \\
-0,2\end{array}$ & $\begin{array}{l}0,560 \\
0,555 \\
0,570\end{array}$ & 210 \\
\hline $\begin{array}{l}14,24 \\
13,64 \\
14,52\end{array}$ & $\begin{array}{l}-4,2 \\
+2,0\end{array}$ & $\begin{array}{l}8,58 \\
7,96 \\
8,43\end{array}$ & $\begin{array}{l}-7,2 \\
-1,7\end{array}$ & $\begin{array}{l}362 \\
333 \\
359\end{array}$ & $\begin{array}{l}-8,0 \\
-0,8\end{array}$ & $\begin{array}{l}42,2 \\
41,8 \\
42,6\end{array}$ & $\begin{array}{l}-0,9 \\
+0,9\end{array}$ & $\begin{array}{l}0,610 \\
0,585 \\
0,600\end{array}$ & $\begin{array}{l}195 \\
190\end{array}$ \\
\hline $\begin{array}{l}12,80 \\
12,52 \\
13,08\end{array}$ & $\begin{array}{l}-2,2 \\
+2,2\end{array}$ & $\begin{array}{l}7,46 \\
7,20 \\
7,85\end{array}$ & $\begin{array}{l}-3,5 \\
+5,2\end{array}$ & $\begin{array}{l}316 \\
290 \\
337\end{array}$ & $\begin{array}{l}-8,2 \\
+6,6\end{array}$ & $\begin{array}{l}42,3 \\
40,3 \\
42,9\end{array}$ & $\begin{array}{l}-4,7 \\
+1,4\end{array}$ & $\begin{array}{l}0,595 \\
0,590 \\
0,595\end{array}$ & 170 \\
\hline $\begin{array}{l}14,00 \\
13,94 \\
15,08\end{array}$ & $\begin{array}{r}-0,4 \\
+7,7\end{array}$ & $\begin{array}{l}7,67 \\
7,53 \\
8,01\end{array}$ & $\begin{array}{r}-1,8 \\
+4,4\end{array}$ & $\begin{array}{l}350 \\
342 \\
369\end{array}$ & $\begin{array}{r}-2,3 \\
+5,4\end{array}$ & $\begin{array}{l}45,6 \\
45,4 \\
46,1\end{array}$ & $\begin{array}{r}-0,4 \\
+1,1\end{array}$ & $\begin{array}{l}0,610 \\
0,605 \\
0,610\end{array}$ & 160 \\
\hline $\begin{array}{l}14,91 \\
14,91 \\
15,02 \\
\end{array}$ & $\begin{array}{l} \pm 0 \\
+0,7\end{array}$ & $\begin{array}{l}7,65 \\
7,46 \\
7,76\end{array}$ & $\begin{array}{l}-2,5 \\
+1,4\end{array}$ & $\begin{array}{l}337 \\
323 \\
342\end{array}$ & $\begin{array}{l}-4,1 \\
+1,5\end{array}$ & $\begin{array}{l}44,0 \\
43,4 \\
44,1\end{array}$ & $\begin{array}{l}-1,4 \\
+0,2\end{array}$ & $\begin{array}{l}0,575 \\
0,570 \\
0,575\end{array}$ & 190 \\
\hline $\begin{array}{l}14,66 \\
14,52 \\
14,66\end{array}$ & $\begin{array}{l}-0,9 \\
\pm 0\end{array}$ & $\begin{array}{l}8,58 \\
8,06 \\
8,45\end{array}$ & $\begin{array}{l}-6,1 \\
-1,5\end{array}$ & $\begin{array}{l}407 \\
375 \\
406\end{array}$ & $\begin{array}{l}-7,9 \\
-0,2\end{array}$ & $\begin{array}{l}47,4 \\
46,5 \\
48,0\end{array}$ & $\begin{array}{l}-1,9 \\
+1,3\end{array}$ & $\begin{array}{l}0,610 \\
0,610 \\
0,605\end{array}$ & $\begin{array}{l}188 \\
178\end{array}$ \\
\hline $\begin{array}{l}11,50 \\
11,14 \\
12,41\end{array}$ & $\begin{array}{l}-3,1 \\
+7,9\end{array}$ & $\begin{array}{l}8,28 \\
8,04 \\
8,28\end{array}$ & $\begin{array}{l}-2,9 \\
\pm 0\end{array}$ & $\begin{array}{l}385 \\
365 \\
380\end{array}$ & $\begin{array}{l}-5,2 \\
-1,3\end{array}$ & $\begin{array}{l}46,5 \\
45,4 \\
45,8\end{array}$ & $\begin{array}{l}-2,4 \\
-1,5\end{array}$ & $\begin{array}{l}0,625 \\
0,615 \\
0,625\end{array}$ & 190 \\
\hline
\end{tabular}

hrte Wasser von $1 \frac{1}{2} 1$ zum grössten Teil noch im Körper zurückbleibt, abnehmen soll, muss ein Teil des Blutes zusammen mit dem aufgenommenen Wasser in den Blut- und Wasserreservoirs verweilt haben. Die Tatsache, dass auch zur selben Zeit das Eiweiss im zirkulierenden Blut in grösseren Mengen als das Hämoglobin vorhanden ist, bringt uns auf die Vermutung, dass das Eiweiss aus Depotorgen mobilisiert worden 
Fig. 3. Veränderungen des Serumeiweisses und dessen kolloid-osmotischen Drucks durch orale Wasserzufuhr (Wasserversuch nach Volhard) bei essentieller Hypertonie (Fall 1).

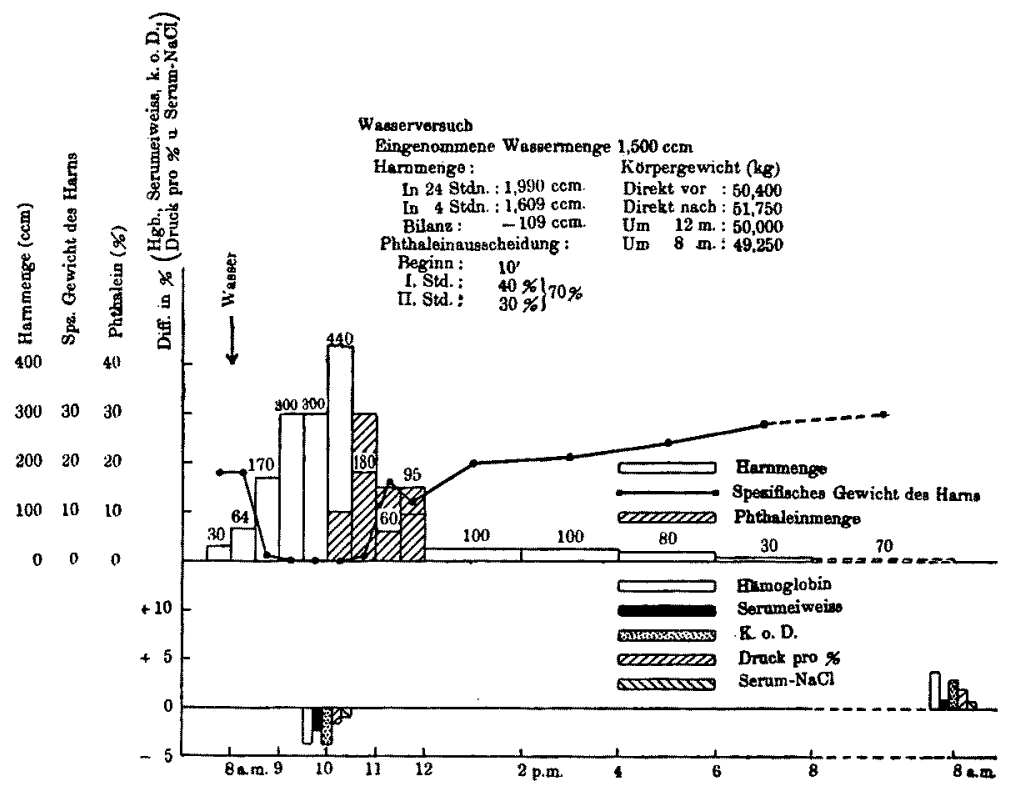

wäre. Hierbei weist der k. o. D. zusammen mit dem Eiweiss das Absinken auf, das aber bei 2 Fällen (Fall 1 und 3) stärker als die Eiweissabnahme erfolgt, während es in einem anderen Fall beinahe in Parallele mit dieser geht. Mithin sinkt der Druck pro \% bei 2 Fällen ab, während er in einem anderen Fall fast unverändert bleibt. Es ist dies höchstwahrscheinlich dahin zu deuten, dass sich in der Regel die Mobilisation von weniger osmoaktiven Eiweissteilchen aus dem Depotorgan findet, dass gelegentlich aber, je nach den Zuständen des Vorrateiweisses im Depotorgan, diejenigen Eiweissteilchen, welche qualitativ den normalen annähernd gleich sind, mobilisiert werden können.

Das oben angeführte Charakteristikum dieser Form, dass die Konzentration des Serumeiweisses grösser als die des Hämoglobins ist, lässt sich auch folgenderweise erklären: Beim Einströmen des zirkulierenden Blutes in das Blutdepot näinlich könnte das besonders konzentriert Erythrozyten enthaltende Blut, mit anderem Wort die Blutzellen mehr als im Verhältnis zum Plasma ins Blutdepot übertreten. Aus diesem Grund wäre es möglich, dass durch das Einströmen des Blutes ins Blutdepot in der Blutbahn das Eiweiss verhältnismässig 
Fig. 4. Veränderungen des Serumeiweisses und dessen kolloid-osmotischen Drucks durch orale Wasserzufuhr (Wasserversuch nach Volhard) bei essentieller Hypertonie (Fall 8).

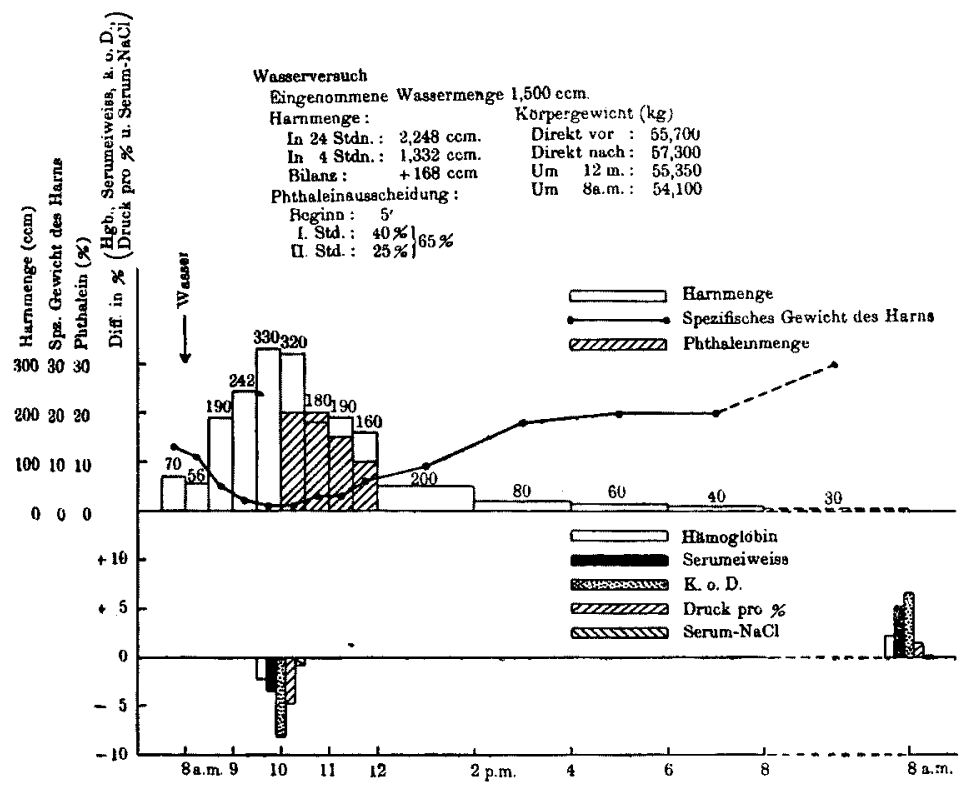

konzentrierter als das Hämoglobin wird. Da aber hierbei das Vorkommen jeglicher Veränderung der Eiweissteilchen im Blut schwer denkbar ist, liegt die Annahme mehr nahe, dass wie eben geschildert, hier relativ grossmolekuläre Eiweissteilchen aus dem Eiweissdepot in die Blutbahn mobilisiert worden sind.

2. Fälle mit geringerer Hämoglobinabnahme und grösserer Eiweissabnahme (Fall 7 und 8): Die Daten über die prozentische $\mathrm{Ab}$ - und Zunahme beim Fall 8 sind beispielweise kurvenmässig in Fig. 4 dargestellt. Bei dieser Form ist die Abnahme des Eiweisses im Verhältnis zum Grad der Blutverdünnung grösser, was auf das teilweise Abströmen des Eiweisses samt dem Wasser ins Gewebe hindeutet. Da der k. o. D. eine grössere Abnahme als die des Eiweisses aufweist, sinkt der Druck pro \% mehr oder weniger ab. Dies dürfte wohl durch die überwiegende Anwesenheit der relativ grösseren Eiweissteilchen im Blut infolge des Abströmens der fein dispersen Eiweissteilchen bedingt sein.

3. Fälle, wo die Hämoglobinabnahme beinahe parallel mit der Eiweissabnahme geht (Fall 6 und 12): Fig. 5 stellt die 
Fig. 5. Veränderungen des Serumeiweisses und dessen kolloid-osmotischen Drucks durch orale Wasserzufuhr (Wasserversuch nach Volhard) bei essentieller Hypertonie (Fall 8).

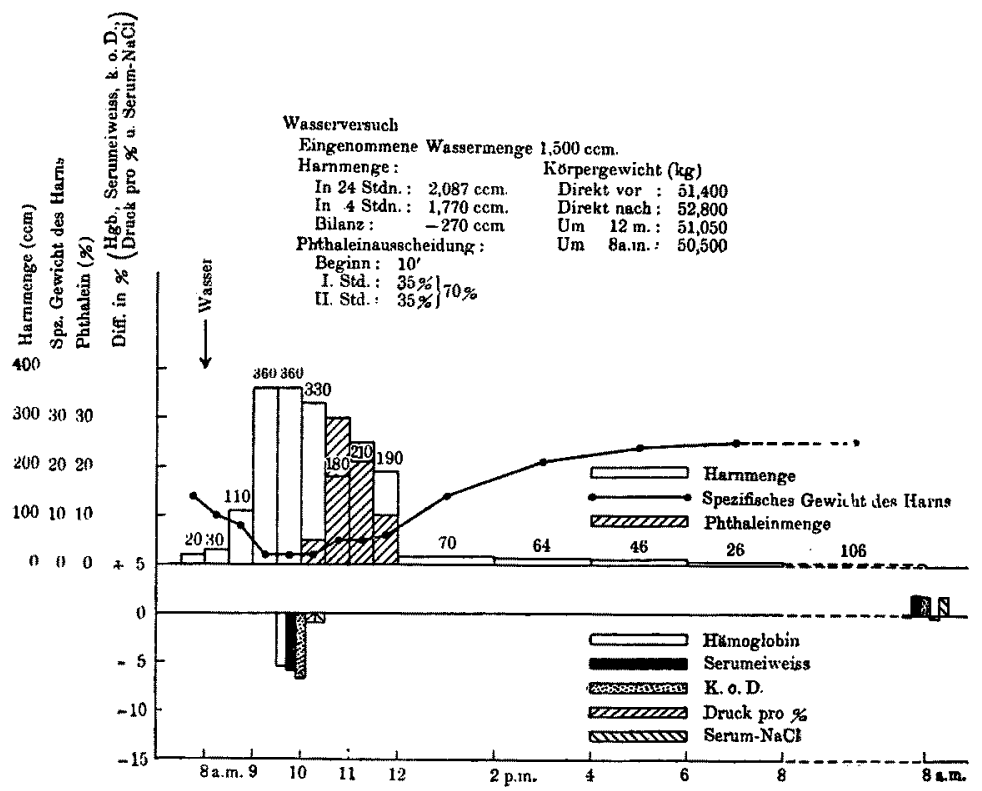

Daten beim Fall 6 in Kurven dar. Bei dieser Form hat man es scheinbar mit dem Austausch des Eiweisses nichts zu tun, da aber der Druck pro \% sich mehr oder weniger erniedrigt hat, dürfte diese Eiweissabnahme nicht als lediglich durch die Blutverdünnung bedingte aufgefasst werden. Wenn man die Verhältnisse bei den oben geschilderten beiden Formen in Erwägung zieht, so dürfte diese Eiweissabnahme darauf beruhen, dass auch hier die Eiweissteilchen teils ins Geweben hineingedrungen, teils aber von dem Depot mobilisiert worden sind.

4. Fälle, wo beim Ausbleiben erheblicher Veränderung in der Hämoglobinkonzentration die Eiweissabnahme erfolgt (Fall 2,5, 9, 10 und 11): Bei vorliegender Form scheint sich die Eiweissmobilisation aus dem Depot noch nicht vollends zu vollziehen, während zu gleicher Zeit ein Teil des Eiweissbestandes des Blutes schon ins Gewebe eingedrungen ist, und aus dem Umstand, dass der k. o. D. sowie der Druck pro \% mehr oder weniger erniedrigte Werte aufweisen, kann geschlossen werden, dass auch die abgeströmten Eiweissteilchen relativ feindisperser Art sind. 
Beim Ưberblick über oben erwähnte Daten bei 4 Formen gewinnt man durchaus den Eindruck, dass jedenfalls letzten Endes zusammen mit der Verschiebung des Blutes und Wassers auch auf der Eiweisseite unter dem Anreiz der Wasserzufuhr stets die Mobilisation der relativ grobdispersen Eiweissteilchen aus dem Depot sowie das Hineindringen der relativ feindispersen Eiweissteilchen in die Gewebe stattfinden, und dass, je nach der Art und Weise dieser Vorgänge, d. h. je nachdem ob die Mobilisation aus dem Depot intensiver oder ob das Hineindringen des Eiweisses in die Gewebe stärker vor sich geht, sich verschiedentliche Formen ergeben.

Kurzum, bei essentieller Hypertonie unterscheidet sich die nach Flüssigkeitszufuhr auftretende Veränderung des Blutes dadurch von derselben bei Gesunden, dass die primäre Eindickung verzögert eintritt, indem das Blut nämlich 2 Stunden nach der Wasserzufuhr noch in der Phase initieller Hydrämie verharrt oder sich noch auf dem Übergangsstadium von initieller Hydrämie zu primärer Eindickung befindet, und das Serumeiweiss an sich verhält sich ganz ähnlich wie bei Gesunden, es handelt sich nämlich hierbei um das Einströmen der relativ grossen Eiweissteilchen aus dem Depot ins Blut und um das Abströmen der relativ kleinen Eiweissteilchen ins Gewebe.

Blutchlorspiegel erhält, abgesehen von einigen Ausnahmefällen mit geringfügigem Absinken, in der überwiegenden Mehrzahl der Fälle annähernde normale Werte.

Was die Veränderungen im Blut nach 24 Stunden anbelangt, schlägt das Hämoglobin sowohl bei Fällen, wo es in 2stündigem Ablauf nach Wasserzufuhr abgenommen hat, wie auch bei denjenigen Fällen, wo es zur selben Stunde unverändert geblieben ist, im allgemeinen den Weg zu mehr oder weniger Zunahme ein. Hierbei ist bei 9 von 12 Fällen der Ursprungswert überschritten, bei restlichen 3 Fällen wird er erreicht. In dieser Zeitspanne weist das Blut also eine terminale Bluteindickung, in wenn auch gewissem Umfang, auf; es fällt indessen auf, dass diese terminale Bluteindickung, verglichen mit derselben bei Gesunden, mannigfache Abstufungen bietet. In diesem Zeitpunkt schlagen das Bluteiweiss und k. o.D. gemeinsam miteinander den Weg zur Wiederherstellung ein, gehen aber nicht immer parallel mit dem Verhalten des Hämoglobins, indem beide erstere verschiedene Deviationen erkennen lassen. In manchen Fällen nämlich ist die Erhöhung des k. o. D. kleiner als die Zunahme des Hämoglobins; weil aber der k. o.D. im ganzen genommen zunimmt, erfährt der Druck pro \% in der Mehrzahl der Fälle eine Erhöhung. Deshalb geht die quantitative 
sowie qualitative Veränderung des Eiweisses hier meistens mit der Konzentrationszunahme des Blutes einher. Bei näherer Beobachtung einzelner Fälle bemerkt man jedoch, dass es manche Fälle gibt, in denen grosse Unterschiede auf dem Verhältnisse der Zunahme der Hämoglobinkonzentration zu der des Serumeiweissgehaltes bestehen (Fall 3, 8 und 12). Dies spricht zweifellos dafür, dass der Eiweissaustausch zwischen Depot und Gewebe auch nach 24 Stunden ebenso wie nach 2 Stunden, in wenn auch abgeschwächtem Masse, vor sich geht. Aus dem Umstand indessen, dass hierbei der Druck pro \% mehr oder weniger erhöht ist, dürfte wohl geschlossen werden, dass im Gegensatz zur Veränderung im 2stündigen Ablauf relativ kleine Eiweissteilchen im Blut sich vermehren.

Der Blutchlorspiegel erreicht in allen Fällen den Normalwert.

Oben auseinandergesetzte Ergebnisse lassen sich wie folgt zusammenfassen: Nach peroraler Zufuhr von grossen Wassermengen bei essentiellen Hypertonikern spielen sich 2 Stunden später die Vorgänge ab, welche im Hineindringen der kleinen Teilchen von Bluteiweiss ins Gewebe und in der Mobilisation der grösseren Eiweissteilchen aus dem Depot ihren Ausdruck finden. Es stellen sich hierbei durch das Überwiegen des einen Vorganges uiber den anderen verschiedene quantitative Veränderungen an Bluteiweisskörpern ein, es kommt aber qualitativ stets zur Zunahme der grösseren Eiweissteilchen, weshalb der k. o.D. sowie der Druck pro \% Erniedrigungen erfahren. In 24 stündigem Ablauf streben das Eiweiss und der k. o.D. im Verein mit der Bluteindickung gemeinsam ständig dem Anfangsniveau zu; weil aber noch in diesem Zeitpunkt der Austausch des Bluteiweisses zwischen Eiweissdepotorgen bzw. Gewebe und Blut in wenn auch gewissem Ausmass anscheinend fortbesteht, geht die quantitative Beziehung zwischen dem Hämoglobin und Eiweiss nicht in jedem Fall in Parallele.

\section{Versuch an Nierenkranken.}

Es wurden als das Krankenmaterial mit gestörter Nierenfunktion 9 Fälle von chronischer Glomerulonephritis ohne erhebliche Niereninsuffizienz, 4 Fälle von sekundärer Schrumpfniere und 3 Fälle von maligner Hypertonie gewählt. Das Ergebnis des an 9 Fällen von chronischen Glomerulonephritiden angestellte Wasserversuchs ergaben gegenüber den an 7 Fällen von 2 letztgenannten Krankheitsgruppen erhobenen Befunden erhebliche Unterschiede, indem der Wasserversuch bei erstgenannten 9 Fällen keine grosse Abweichung 
von derNorm darbot, während es er bei letzteren 7 Fällen auf manifeste Niereninsuffizienz hingewiesen hat. Aus Rücksicht darauf seien im folgenden die Versuchsdaten unter Einteilung in die Gruppe A und B angeführt.

\section{A. Chronische Glomerulonephritiden ohne erhebliche Niereninsuffizienz.}

Die Resultate des an 9 Glomerulonephritiden angestellten Wasserversuchs mit Blutuntersuchungen sind in Tab. 3 wiedergegeben. Die Resultate des Wasserversuchs seien nachfolgend in aller Kürze erwähnt. Die Wasserausscheidung war zeitlich verzögert, die in den ersten 4 Stunden ausgeschiedene Harnmenge war selbstverständlich geringer sowohl als bei Gesunden wie auch bei Hypertonikern. Die Wasserbilanz wurde, abgesehen von 2 Ausnahmefällen mit negativer Bilanz, bei übrigen 7 Fällen deutlich positiv. Wie aus Tab. 3 ersichtlich ist, war das Verdünnungs- und Konzentrationsvermögen in geringem Masse gestört. Die Phthaleinausscheidung war annähernd normal. Die Wasserausscheidung sowie das Verdünnungs-und Konzentrationsvermögen waren also in wenn auch geringem Ausmasse unverkennbar gestört.

Betreffend die 2 Stunden nach der Wasserzufuhr aufgetretenen Veränderungen des Blutes, war das Hämoglobin ohne Ausnahme herabgesetzt. In sämtlichen Fällen wurde nämlich hier noch die initiale Hydrämie angetroffen. Was das Verhalten des Bluteiweisses in dieser Zeitspanne anbelangt, wurden hier ausschliesslich erniedrigte Werte gefunden. Wenn man indessen die prozentuelle Abnahme des Hämoglobins mit der des Eiweisses vergleicht, so ergibt sich daraus, dass von sätmlichen 9 Fällen bei 6 Fällen (Fall 1,2,3,6,7 und 8) die prozentuelle Abnahme des Eiweisses kleiner als die des Hämoglobins ist, während sie bei übrigen 3 Fällen (Fall 4, 5 und 9) hingegen grösser als die letztere ist. Aus demselben Gesichtspunkt, wie er im vorangehenden Kapitel geäussert wurde, dürfte auch angenommen werden, dass im ersteren Fall die Abnahme des Eiweisses wegen erfolgter Eiweissmobilisation aus dem Depot im Vergleich zur Blutverdünnung kleiner ist, während im letzteren Fall die Abnahme des Eiweisses im Verhältnis zum Grad der Blutverdünnung deshalb in stärkerem Masse zustande kommt, weil das Serumeiweiss zum Teil ins Gewebe hineingegangen ist.

Der k. o. D. sinkt abgesehen von einem Ausnahmefall (Fall 3), in allen übrigen Fällen ab, und da dieser Abfall verhältnismässig grösser 
Tabelle

Versuch an chronischen

\begin{tabular}{|c|c|c|c|c|c|c|c|c|c|}
\hline \multirow[b]{3}{*}{ Fall } & \multirow{3}{*}{$\begin{array}{c}\text { Name, } \\
\text { Alter } \\
\text { u. } \\
\text { Gesch- } \\
\text { lecht }\end{array}$} & \multirow{3}{*}{$\begin{array}{l}\text { Harn- } \\
\text { menge } \\
\text { in } 24 \\
\text { Stdn. } \\
\text { (ccm) }\end{array}$} & \multicolumn{4}{|c|}{ Resultate $\mathrm{d}$. Wasserversuchs } & \multirow[b]{3}{*}{$\begin{array}{c}\text { Zeit d. } \\
\text { Blut- } \\
\text { entnahme } \\
\text { (Std.) }\end{array}$} & \multirow{2}{*}{\multicolumn{2}{|c|}{ Hämoglobin }} \\
\hline & & & \multirow[b]{2}{*}{$\begin{array}{c}\text { Bilanz } \\
\text { in } 4 \\
\text { Stdn. } \\
(\operatorname{ccm})\end{array}$} & \multirow[b]{2}{*}{$\begin{array}{l}\text { Min. u. max } \\
\text { Sp. Gewicht } \\
\text { d. Urins }\end{array}$} & \multicolumn{2}{|c|}{ Phthaleinprobe } & & & \\
\hline & & & & & $\begin{array}{c}\text { Beginn } \\
\text { (Min.) }\end{array}$ & $\begin{array}{c}\text { Innerhalb } \\
2 \text { Stdn. } \\
\text { ausgeschie- } \\
\text { dene Menge } \\
(\%)\end{array}$ & & $\mathrm{g} / \mathrm{dl}$ & $\begin{array}{l}\text { Diff. } \\
\text { in } \\
\%\end{array}$ \\
\hline 1 & $\begin{array}{c}\text { S. K. } \\
32 \\
\text { m }\end{array}$ & 1,718 & +372 & $1,002-1,026$ & 10 & 60 & $\begin{array}{ll}\text { Vor } & \\
\text { Nach } & 2 \\
\quad & 24\end{array}$ & $\begin{array}{l}14,24 \\
13,82 \\
14,10\end{array}$ & $\begin{array}{l}-2,9 \\
-1,0\end{array}$ \\
\hline 2 & $\begin{array}{c}\text { R. S. } \\
\mathbf{5 0} \\
\mathbf{w}\end{array}$ & 1,528 & +208 & $1,001-1,025$ & 10 & 75 & $\begin{array}{ll}\text { Vor } & \\
\text { Nach } & 2 \\
\Rightarrow & 24\end{array}$ & $\begin{array}{l}13,64 \\
13,08 \\
13,78\end{array}$ & $\begin{array}{r}-4,1 \\
+1,0\end{array}$ \\
\hline 3 & $\begin{array}{l}\text { M. T. } \\
20 \\
\text { m }\end{array}$ & 1,664 & +120 & $1,000-1,031$ & 10 & 55 & $\begin{array}{ll}\text { Vor } & \\
\text { Nach } & 2 \\
" & 24\end{array}$ & $\begin{array}{l}17,08 \\
16,48 \\
17,08\end{array}$ & $\begin{array}{l}-3,5 \\
\pm 0\end{array}$ \\
\hline 4 & $\begin{array}{c}\text { K. I. } \\
42 \\
\text { w }\end{array}$ & 1,741 & +142 & $1,001-1,022$ & 10 & 50 & $\begin{array}{ll}\text { Vor } & \\
\text { Nach } & 2 \\
\quad & 24\end{array}$ & $\begin{array}{l}11,92 \\
11,25 \\
12,24\end{array}$ & $\begin{array}{l}-5,6 \\
+2,7\end{array}$ \\
\hline 5 & $\begin{array}{c}\text { T. H. } \\
49 \\
\text { m }\end{array}$ & 1,530 & +580 & $1,001-1,025$ & 10 & 60 & $\begin{array}{lr}\text { Vor } & \\
\text { Nach } & 2 \\
\Rightarrow & 24\end{array}$ & $\begin{array}{l}14,63 \\
14,24 \\
14,08\end{array}$ & $\begin{array}{l}-2,7 \\
-3,7\end{array}$ \\
\hline 6 & $\begin{array}{c}\text { Z. } 0 . \\
29 \\
\text { m }\end{array}$ & 2,148 & -104 & $1,002-1,024$ & 10 & 65 & $\begin{array}{ll}\text { Vor } & \\
\text { Nach } & 2 \\
" & 24\end{array}$ & $\begin{array}{l}13,84 \\
13,25 \\
13,94\end{array}$ & $\begin{array}{l}-4,3 \\
+0,7\end{array}$ \\
\hline 8 & $\begin{array}{c}\text { T. S. } \\
36 \\
w\end{array}$ & 1,792 & +144 & $1,003-1,032$ & 10 & 60 & $\begin{array}{ll}\text { Vor } & \\
\text { Nach } & 2 \\
\Rightarrow & 24\end{array}$ & $\begin{array}{r}10,32 \\
9,96 \\
9,68\end{array}$ & $\begin{array}{l}-3,5 \\
-6,2\end{array}$ \\
\hline 8 & $\begin{array}{c}\text { S. E. } \\
52 \\
\text { w }\end{array}$ & 1,916 & -64 & $1,004-1,032$ & 10 & 70 & $\begin{array}{ll}\text { Vor } & \\
\text { Nach } & 2 \\
\Rightarrow & 24\end{array}$ & $\begin{array}{l}14,86 \\
14,38 \\
14,49\end{array}$ & $\begin{array}{l}-3,2 \\
-2,5\end{array}$ \\
\hline 9 & $\begin{array}{c}\text { H. E. } \\
53 \\
w\end{array}$ & 1,910 & +110 & $1,001-1,024$ & 10 & 65 & $\begin{array}{ll}\text { Vor } & \\
\text { Nach } & 2 \\
\quad, & 24\end{array}$ & $\begin{array}{l}16,06 \\
15,78 \\
15,36\end{array}$ & $\begin{array}{l}-1,7 \\
-4,3\end{array}$ \\
\hline
\end{tabular}

als die Abnahme des Eiweisses ist, erfährt der Druck pro \% ausnahmslos eine Erniedrigung. Daraus geht zweifellos hervor, dass es sich hierbei um die Mobilisierung der relativ grossförmigen Eiweissteilchen und um den Übertritt der relativ kleinförmigen Eiweissteilchen aus der Blutbahn ins Gewebe handelt. Oben sind die Fälle von zweierlei Art, d. h. die Fälle mit vorwiegender Eiweissmobilisierung aus dem Depot 
3.

Glomerulonephritiden.

\begin{tabular}{|c|c|c|c|c|c|c|c|c|}
\hline \multicolumn{7}{|c|}{ Blutuntersuehung } & \multirow{3}{*}{$\begin{array}{c}\text { Blut- } \\
\text { druck } \\
(\mathrm{mm} \\
\mathrm{Hg})\end{array}$} & \multirow[b]{3}{*}{ Bemerkung } \\
\hline \multicolumn{2}{|c|}{ Serumeiweiss } & \multicolumn{2}{|c|}{ K. o. D. } & \multicolumn{2}{|c|}{ Druck pro $\%$} & \multirow[b]{2}{*}{$\begin{array}{l}\text { Serum- } \\
\mathrm{NaCl} \\
(\mathrm{g} / \mathrm{dl})\end{array}$} & & \\
\hline$\%$ & $\begin{array}{l}\text { Diff. } \\
\text { in } \\
\%\end{array}$ & $\begin{array}{l}\mathrm{mm} \\
\mathrm{H}_{2} \mathrm{O}\end{array}$ & $\begin{array}{l}\text { Diff. } \\
\text { in } \\
\%\end{array}$ & $\begin{array}{l}\mathrm{mm} \\
\mathrm{H}_{2} \mathrm{O}\end{array}$ & $\begin{array}{l}\text { Diff. } \\
\text { in } \\
\%\end{array}$ & & & \\
\hline $\begin{array}{l}8,62 \\
8,47 \\
8,77\end{array}$ & $\begin{array}{l}-2,3 \\
+1,1\end{array}$ & $\begin{array}{l}381 \\
349 \\
382\end{array}$ & $\begin{array}{l}-8,4 \\
+0,3\end{array}$ & $\begin{array}{l}43,9 \\
41,2 \\
43,5\end{array}$ & $\begin{array}{l}-6,1 \\
-0,9\end{array}$ & $\begin{array}{l}0,580 \\
0,555 \\
0,565\end{array}$ & $\begin{array}{l}128 \\
100\end{array}$ & $\begin{array}{l}\text { Harn: Eiweiss } 1,5 \% \text {, Rote } \\
\text { mässig, Zylinder spärlich. } \\
\text { Spur Ödem an Tibiakante. }\end{array}$ \\
\hline $\begin{array}{l}8,69 \\
8,45 \\
8,96\end{array}$ & $\begin{array}{l}-2,8 \\
+3,1\end{array}$ & $\begin{array}{l}390 \\
372 \\
405\end{array}$ & $\begin{array}{l}-4,5 \\
+3,8\end{array}$ & $\begin{array}{l}44,9 \\
44,0 \\
45,2\end{array}$ & $\begin{array}{l}-2,0 \\
+0,7\end{array}$ & $\begin{array}{l}0,615 \\
0,605 \\
0,615\end{array}$ & $\begin{array}{l}195 \\
210\end{array}$ & $\begin{array}{l}\text { Harn : Eiweiss 1,5\%, Rote } \\
\text { spärlich. Blut-RN } 27,7 \\
\text { mg/dl. }\end{array}$ \\
\hline $\begin{array}{l}8,28 \\
8,13 \\
8,42\end{array}$ & $\begin{array}{l}-1,8 \\
+1,7\end{array}$ & $\begin{array}{l}382 \\
385 \\
404\end{array}$ & $\begin{array}{l}+0,8 \\
+5,7\end{array}$ & $\begin{array}{l}47,3 \\
47,3 \\
48,0\end{array}$ & $\begin{array}{l} \pm 0 \\
+1,5\end{array}$ & $\begin{array}{l}0,595 \\
0,592 \\
0,595\end{array}$ & $\begin{array}{l}135 \\
135\end{array}$ & $\begin{array}{l}\text { Harn : Eiweiss schwach, } \\
\text { Rote u. Zylinder neg. } \\
\text { Blut-RN } 29,5 \mathrm{mg} / \mathrm{dl} \text {. }\end{array}$ \\
\hline $\begin{array}{l}7,22 \\
6,77 \\
7,46\end{array}$ & $\begin{array}{l}-6,2 \\
+3,3\end{array}$ & $\begin{array}{l}310 \\
284 \\
314\end{array}$ & $\begin{array}{l}-8,4 \\
+1,3\end{array}$ & $\begin{array}{l}42,9 \\
41,9 \\
42,1\end{array}$ & $\begin{array}{l}-2,3 \\
-1,9\end{array}$ & $\begin{array}{l}0,545 \\
0,545 \\
0,545\end{array}$ & 144 & $\begin{array}{l}\text { Harn : Eiweiss Spur. Blut- } \\
\text { RN } 34,84 \mathrm{mg} / \mathrm{dl} \text {. }\end{array}$ \\
\hline $\begin{array}{l}7,27 \\
6,81 \\
7,31\end{array}$ & $\begin{array}{l}-6,3 \\
+0,5\end{array}$ & $\begin{array}{l}277 \\
251 \\
267\end{array}$ & $\begin{array}{l}-9,4 \\
-3,6\end{array}$ & $\begin{array}{l}38,1 \\
36,8 \\
36,5\end{array}$ & $\begin{array}{l}-3,4 \\
-4,2\end{array}$ & $\begin{array}{l}0,525 \\
0,515 \\
0,535\end{array}$ & $\begin{array}{l}116 \\
115\end{array}$ & $\begin{array}{l}\text { Harn : Eiweiss u. Rote } \\
\text { mässig. Blut-RN } 31,5 \\
\text { mg/dl. Oddem am Unter- } \\
\text { schenkel deutlich. }\end{array}$ \\
\hline $\begin{array}{l}5,88 \\
5,79 \\
6,08\end{array}$ & $\begin{array}{l}-1,4 \\
+3,4\end{array}$ & $\begin{array}{l}215 \\
202 \\
226\end{array}$ & $\begin{array}{l}-6,0 \\
+5,2\end{array}$ & $\begin{array}{l}36,4 \\
34,9 \\
37,2\end{array}$ & $\begin{array}{l}-4,1 \\
+2,2\end{array}$ & $\begin{array}{l}0,585 \\
0,580 \\
0,587\end{array}$ & 135 & $\begin{array}{l}\text { Harn: Eiweiss } 3 \% \text {, Rote } \\
\text { mässig, aber Zylinder neg. } \\
\text { Spur ödem am Bein. Blut- } \\
\text { RN } 43,4 \mathrm{mg} / \mathrm{dl} \text {. }\end{array}$ \\
\hline $\begin{array}{l}7,74 \\
7,53 \\
7,53\end{array}$ & $\begin{array}{l}-2,7 \\
-2,7\end{array}$ & $\begin{array}{l}301 \\
286 \\
300\end{array}$ & $\begin{array}{l}-5,0 \\
-0,3\end{array}$ & $\begin{array}{l}38,9 \\
38,0 \\
39,9\end{array}$ & $\begin{array}{l}-2,3 \\
+2,6\end{array}$ & $\begin{array}{l}0,575 \\
0,570 \\
0,575\end{array}$ & $\begin{array}{r}100 \\
92\end{array}$ & $\begin{array}{l}\text { Harn: Eiweiss } 1 \% \text {, Rote } \\
\text { u. Zylinder spärlich. }\end{array}$ \\
\hline $\begin{array}{l}8,32 \\
8,10 \\
8,08\end{array}$ & $\begin{array}{l}-2,6 \\
-2,9\end{array}$ & $\begin{array}{l}419 \\
393 \\
396\end{array}$ & $\begin{array}{l}-6,2 \\
-1,7\end{array}$ & $\begin{array}{l}50,4 \\
48,5 \\
49,0\end{array}$ & $\begin{array}{l}-3,7 \\
-2,8\end{array}$ & $\begin{array}{l}0,630 \\
0,595 \\
0,620\end{array}$ & $\begin{array}{l}130 \\
132\end{array}$ & $\begin{array}{c}\text { Harn: Rote u. Zylinder } \\
\text { spärlich. Kein Ödem. }\end{array}$ \\
\hline $\begin{array}{l}8,33 \\
8,06 \\
8,17\end{array}$ & $\begin{array}{l}-3,2 \\
-1,9\end{array}$ & $\begin{array}{l}377 \\
360 \\
368\end{array}$ & $\begin{array}{l}-4,5 \\
-2,4\end{array}$ & $\begin{array}{l}45,2 \\
44,4 \\
45,0\end{array}$ & $\begin{array}{l}-1,8 \\
-0,4\end{array}$ & $\begin{array}{l}0,610 \\
0,610 \\
0,600\end{array}$ & $\begin{array}{l}125 \\
120\end{array}$ & $\begin{array}{l}\text { Harn: Spur Eiweiss, Rote } \\
\text { spärlich. }\end{array}$ \\
\hline
\end{tabular}

und die Fälle mit vorwiegendem Übertritt des Eiweisses aus dem Blut ins Gewebe gesondert erwähnt worden. Da bei näherer Analysierung einzelner Fälle solche Fälle, welche eine Übergangsform zwischen den beiden Typen darzustellen scheinen, anzutreffen sind, dürfte die Annahme wohl zu Recht bestehen, dass 2 Stunden nach der Wasserzufuhr, wie oben bereits geschildert, eben angedeutete Austauschvorgänge von 
Fig. 6. Veränderungen des Serumeiweisses und dessen kolloid-osmotischen Drucks durch orale Wasserzufuhr (Wasserversuch nach Volhard) bei Glomerulonephritis diffusa chronica (Fall 1).

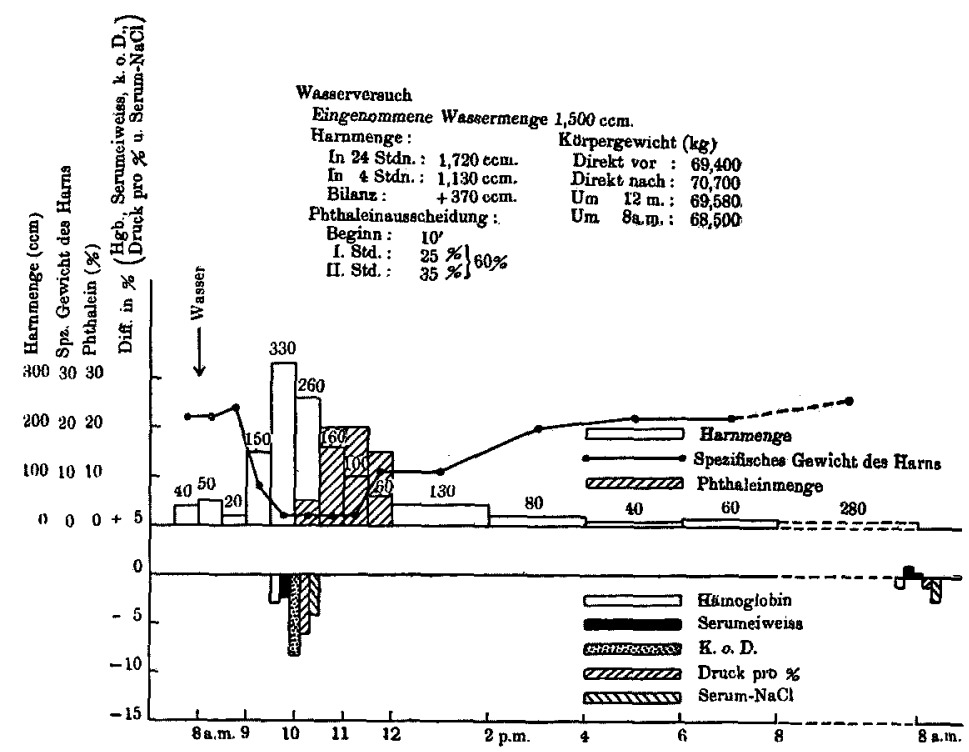

zweierlei Art zugleich stattfinden; und dass durch das Úberwiegen des einen Vorgangs über den anderen verschiedentliche Form resultiert.

Blutchlorspiegel ist im allgemeinen herabgesetzt, jedoch in sehr geringem Masse; beim Auftreten der Blutverdünnung scheint ein Teil des Gewebskochsalzes ins Blut überzugehen, damit der NaCl-Spiegel des Blutes auf einer bestimmten Höhe erhalten werden kann.

Figg. 6 und 7 geben die Daten bei Fall 1 resp. 5 als Versuchsbeispiel in Kurven wieder.

In 24stündigem Ablauf schlägt die Hämoglobinkonzentration in 6 von 9 Fällen (Fall 1, 2, 3, 4, 6 und 8) gegenüber den 2Stundenwerten den Weg zur Zunahme, in wenn auch gewissen Ausmasse, ein. Allein *selbst bei Fällen, wo eine erhebliche Vermehrung auftritt, bewegt sich das Hämoglobin um den Vorwert herum. Die Intensität der terminalen Bluteindickung zur selben Stunde wird also nicht nur von derselben bei Gesunden weitgehend übertroffen, sondern ist auch geringer als bei Hypertonikern. In übrigen 3 Fällen (Fall 5, 7 und 9) tritt jegliche terminale Eindickung nicht ein, so dass der Hämoglobinwert gegenüber dem 2stündigem Wert eher herabgesetzt ist.

Eiweiss wird, mit Ausnahme von 1 oder 2 Fällen, in allen übri- 
Fig. 7. Veränderungen des Serumeiweisses und dessen kolloid-osmotischen Drucks durch orale Wasserzufuhr (Wasserversuch nach Volhard) bei Glomerulonephritis diffusa chronica (Fall 5).

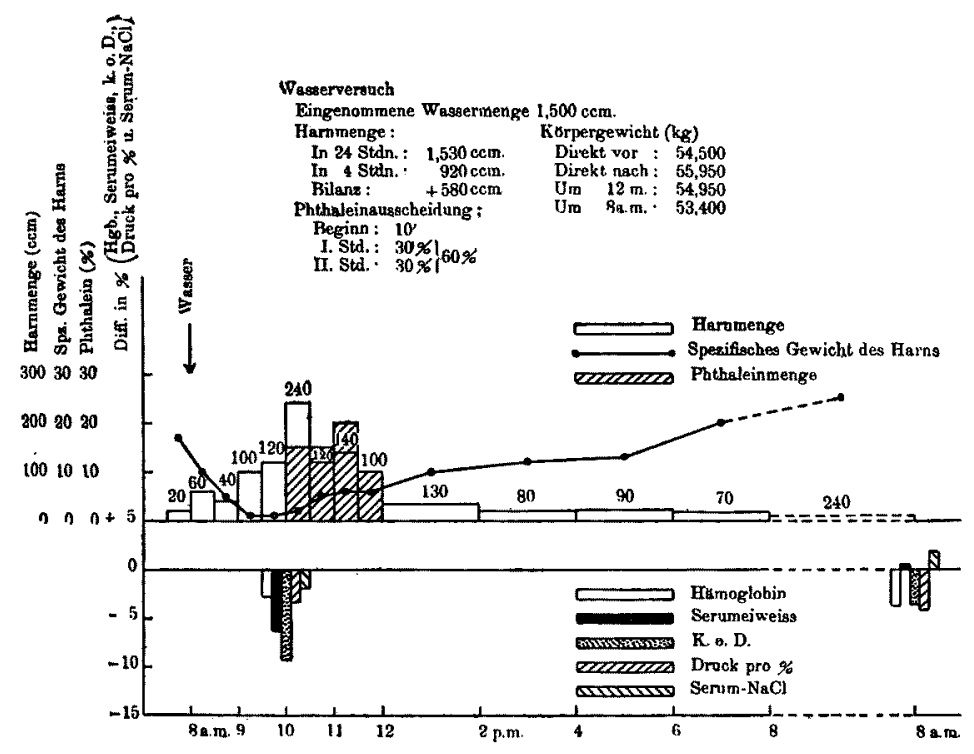

gen Fällen nach 24 Stunden mehr oder weniger vermehrt gefunden. K. o. D. erfährt auch eine Erhöhung, der Druck pro \% erhöht sich, ausgenommen 1 Fall, in übrigen 8 Fällen gewissermassen, und zwar sind die Grade dieser Erhöhung im Vergleich mit Gesunden und $\mathrm{Hy}$ pertonikern im allgemeinen geringer. Der NaCl-Spiegel zeigt im grossen ganzen keine bemerkbare Veränderung.

B. Fälle mit manifester Niereninsuffizienz (sekundäre Schrumpfniere und maligne Hypertonie).

Nach Ergebnissen des Wasserversuchs ist meines Erachtens nicht nötig, die Ergebnisse bei den Kranken an sekundärer Schrumpfniere und bei den malignen Hypertonikern gesondert einer Betrachtung zu unterziehen, weil hochgradige Störungen der Nierenfunktion den beiden gemeinsam waren. Deshalb sind Resultate des an 4 Fällen von sekundärer Schrumpfniere sowie an 3 Fällen von maligner Hypertonie ausgeführten Wasserversuchs wie auch der Blutuntersuchungen beide zusammen in Tab. 4 angegeben. 
Tabelle

Versuch an maligner Hypertonie

\begin{tabular}{|c|c|c|c|c|c|c|c|c|c|}
\hline \multirow[b]{3}{*}{ Fall } & \multirow{3}{*}{$\begin{array}{l}\text { Name, } \\
\text { Alter } \\
\text { u. } \\
\text { Gesch- } \\
\text { lecht. }\end{array}$} & \multicolumn{5}{|c|}{ Resultate d. Wasserversuchs } & \multirow[b]{3}{*}{$\begin{array}{l}\text { Zeit d. } \\
\text { Blutent- } \\
\text { nahme } \\
\text { (Std.) }\end{array}$} & & \\
\hline & & \multirow{2}{*}{$\begin{array}{l}\text { Harn- } \\
\text { menge } \\
\text { in } 24 \\
\text { Stdn. } \\
\text { (ccm) }\end{array}$} & \multirow[b]{2}{*}{$\begin{array}{c}\text { Bilanz } \\
\text { in } 4 \\
\text { Stdn. } \\
(\mathrm{ccm})\end{array}$} & \multirow[b]{2}{*}{$\begin{array}{l}\text { Min. u. } \\
\text { max. Sp. } \\
\text { Gewicht } \\
\text { d. Urins }\end{array}$} & \multicolumn{2}{|c|}{ Phthaleinprobe } & & \multicolumn{2}{|c|}{ Hämoglobin } \\
\hline & & & & & $\begin{array}{l}\text { Beginn } \\
\text { (Min.) }\end{array}$ & $\begin{array}{l}\text { Innerhalb } \\
2 \text { Stdn. } \\
\text { ausgeschie- } \\
\text { dene Menge } \\
(\%)\end{array}$ & & $\mathrm{g} / \mathrm{dl}$ & $\begin{array}{l}\text { Diff. } \\
\text { in } \\
\%\end{array}$ \\
\hline 1 & $\begin{array}{c}\text { K. A. } \\
42 \\
\text { w }\end{array}$ & 1,590 & +860 & $1,004-1,022$ & 15 & 45 & $\begin{array}{ll}\text { Vor } & \\
\text { Nach } & 2 \\
& \quad 24\end{array}$ & $\begin{array}{l}12,24 \\
12,18 \\
11,66\end{array}$ & $\begin{array}{l}-0,5 \\
-4,7\end{array}$ \\
\hline 2 & $\begin{array}{c}\text { M. S. } \\
58 \\
\text { m }\end{array}$ & 936 & +723 & $1,000-1,019$ & 15 & 35 & $\begin{array}{ll}\text { Vor } & \\
\text { Nach } & 2 \\
\Rightarrow & 24\end{array}$ & $\begin{array}{l}9,68 \\
9,96 \\
9,51\end{array}$ & $\begin{array}{r} \\
+2,9 \\
-1,7\end{array}$ \\
\hline 3 & $\begin{array}{c}\text { C. H. } \\
53 \\
\text { m }\end{array}$ & 2,044 & +428 & $1,005-1,020$ & 15 & 40 & $\begin{array}{ll}\text { Vor } & \\
\text { Nach } & 2 \\
, & 24\end{array}$ & $\begin{array}{l}14,52 \\
14,52 \\
14,24\end{array}$ & $\begin{array}{r} \pm 0 \\
-1,9\end{array}$ \\
\hline 4 & $\begin{array}{c}\text { J. A. } \\
\mathbf{5 5} \\
\mathrm{m}\end{array}$ & 1,402 & $+1,225$ & $1,006-1,006$ & 10 & 50 & $\begin{array}{ll}\begin{array}{ll}\text { Vor } & \\
\text { Nach } & 2 \\
& 24\end{array}\end{array}$ & $\begin{array}{l}10,80 \\
10,80 \\
10,52\end{array}$ & $\begin{array}{l} \pm 0 \\
-2,8\end{array}$ \\
\hline 5 & S. K. & 1,871 & +942 & $1,003-1,013$ & 15 & 25 & 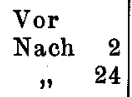 & $\begin{array}{l}8,24 \\
8,52 \\
7,96\end{array}$ & $\begin{array}{l}+3,4 \\
-3,4\end{array}$ \\
\hline 6 & $\begin{array}{c}\text { H. A. } \\
20 \\
\text { w }\end{array}$ & 1,643 & +940 & $1,003-1,015$ & 10 & 35 & $\begin{array}{ll}\text { Vor } & \\
\text { Nach } & 2 \\
\quad, & 24\end{array}$ & $\begin{array}{l}10,46 \\
10,52 \\
10,13\end{array}$ & $\begin{array}{l}+0,6 \\
-3,1\end{array}$ \\
\hline 7 & $\begin{array}{c}\text { M. K. } \\
37 \\
\text { m }\end{array}$ & $\stackrel{\bullet}{1,542}$ & +948 & $1,010-1,018$ & 15 & 30 & $\begin{array}{lr}\text { Vor } & \\
\text { Nach } & 2 \\
\quad " & 24\end{array}$ & $\begin{array}{r}10,30 \\
10,46 \\
9,76\end{array}$ & $\begin{array}{l}+1,5 \\
-5,2\end{array}$ \\
\hline
\end{tabular}

In allen diesen Fällen ist die Wasseranscheidung beträchtlich gestört, die Wasserbilanz in 4 Stunden ist ausschliesslich hochgradig positiv, derart, dass sie zwischen +688 bis $1,225 \mathrm{ccm}$ schwankt. Die Phthaleinausscheidung ist auch stark behindert, das Verdünnungs-und Konzentrationsvermögen ist sehr schlecht, und zwar im gewaltigen Unterschied von oben erwähnten Fällen von chronischer Glomerulonephritis. Diese Verhältnisse veranschaulichen die beigegebenen Kurven (Figg. 8 und 9).

Nach 2 Stunden zeigte die Hämoglobinkonzentration fast keine Veränderung in 4 Fällen (Fall 1, 3,4 und 6) und etliche Zunahme in 3 Fällen (Fall 2, 5 und 7). In 2stiundigem Ablauf nach der Wasser- 
4.

und sekundärer Schrumpfniere.

\begin{tabular}{|c|c|c|c|c|c|c|c|c|}
\hline \multicolumn{7}{|c|}{ Blutuntersuchung } & \multirow{3}{*}{$\begin{array}{c}\text { Blut- } \\
\text { druck } \\
(\mathrm{mm} \\
\mathrm{Hg})\end{array}$} & \multirow[b]{3}{*}{ Bemerkung } \\
\hline \multicolumn{2}{|c|}{ Serumeiweiss } & \multicolumn{2}{|c|}{ K. o.D. } & \multicolumn{2}{|c|}{ Druck pro $\%$} & \multirow[b]{2}{*}{$\begin{array}{l}\text { Serum- } \\
\mathrm{NaCl} \\
(\mathrm{g} / \mathrm{dl})\end{array}$} & & \\
\hline$\%$ & $\begin{array}{l}\text { Diff. } \\
\text { in } \\
\% \%\end{array}$ & $\mathrm{~mm}_{\mathrm{H}_{2} \mathrm{O}}$ & $\begin{array}{l}\text { Diff. } \\
\text { in } \\
\%\end{array}$ & $\begin{array}{l}\mathrm{mm} \\
\mathrm{H}_{2} \mathrm{O}\end{array}$ & $\begin{array}{l}\text { Diff. } \\
\text { in } \\
\%\end{array}$ & & & \\
\hline $\begin{array}{l}8,24 \\
8,15 \\
7,89\end{array}$ & $\begin{array}{l}-1,1 \\
-4,2\end{array}$ & $\begin{array}{l}371 \\
344 \\
344\end{array}$ & $\begin{array}{r}-7,3 \\
-7,3\end{array}$ & $\begin{array}{l}45,1 \\
42,2 \\
43,6\end{array}$ & $\begin{array}{l}-6,4 \\
-3,3\end{array}$ & $\begin{array}{l}0,527 \\
0,525 \\
0,525\end{array}$ & $\begin{array}{l}185 \\
205\end{array}$ & $\begin{array}{l}\text { Maligne Hypertonic, } \\
\text { Harn: Eiweiss schwach, Rote spärlich, } \\
\text { Zylinder neg. Retinitis nephritica. Blut- } \\
\text { RN } 40,1 \mathrm{mg} / \mathrm{dl} \text {. }\end{array}$ \\
\hline $\begin{array}{l}7,80 \\
7,93 \\
7,63\end{array}$ & $\begin{array}{l}+1,7 \\
-2,2\end{array}$ & $\begin{array}{l}288 \\
270 \\
268\end{array}$ & $\begin{array}{r}-6,2 \\
-6,9\end{array}$ & $\begin{array}{l}36,9 \\
34,0 \\
35,1\end{array}$ & $\begin{array}{l}-7,8 \\
-4,9\end{array}$ & $\begin{array}{l}0,520 \\
0,520 \\
0,520\end{array}$ & $\begin{array}{l}180 \\
175\end{array}$ & $\begin{array}{l}\text { Maligne Hypertonie. } \\
\text { Harn: Eiweiss mässig, Rote u. Zylinder } \\
\text { spärlich. Augenfundus: Blutung u. } \\
\text { weissc Flecke. }\end{array}$ \\
\hline $\begin{array}{l}7,39 \\
7,63 \\
7,22\end{array}$ & $\begin{array}{l}+3,2 \\
-2,3\end{array}$ & $\begin{array}{l}296 \\
290 \\
273\end{array}$ & $\begin{array}{r}-2,0 \\
-\quad 7,8\end{array}$ & $\begin{array}{l}40,0 \\
38,0 \\
37,8\end{array}$ & $\begin{array}{l}-5,0 \\
-5,5\end{array}$ & $\begin{array}{l}0,585 \\
0,575 \\
0,580\end{array}$ & 225 & $\begin{array}{l}\text { Maligne Hypertonic. } \\
\text { Harn: Eiweiss 1,2\%o, Rote mässig. } \\
\text { Augenhintergrund: Blutung u. weisse } \\
\text { Flecke. Blut-RN 31, } 7 \text { mg;dl. }\end{array}$ \\
\hline $\begin{array}{l}6,70 \\
6,62 \\
6,23\end{array}$ & $\begin{array}{l}-1,2 \\
-7,0\end{array}$ & $\begin{array}{l}243 \\
231 \\
218\end{array}$ & $\begin{array}{r}-4,9 \\
-10,3\end{array}$ & $\begin{array}{l}36,3 \\
34,9 \\
35,0\end{array}$ & $\begin{array}{l}-3,8 \\
-3,6\end{array}$ & $\begin{array}{l}0,615 \\
0,615 \\
0,612\end{array}$ & $\begin{array}{l}120 \\
100\end{array}$ & $\begin{array}{l}\text { Sekundäre Schrumpfnicre. } \\
\text { Harn: Eiveiss mässigigote Rote u. Zylinder } \\
\text { spärlich. Blut-RN } 81,2 \text { mg/dl. }\end{array}$ \\
\hline $\begin{array}{l}6,75 \\
6,85 \\
6,55\end{array}$ & $\begin{array}{l}+1,5 \\
-3,0\end{array}$ & $\begin{array}{l}264 \\
260 \\
255\end{array}$ & $\begin{array}{r}-1,5 \\
-3,4\end{array}$ & $\begin{array}{l}39,1 \\
37,9 \\
38,8\end{array}$ & $\begin{array}{l}-3,1 \\
-0,8\end{array}$ & $\begin{array}{l}0,560 \\
0,557 \\
0,550\end{array}$ & 205 & $\begin{array}{l}\text { Sekundäre Schrumpfnicre. Zuweilen Ödem } \\
\text { an Bein. Neuroretinitis nephritica. } \\
\text { Harn: Eivweis } 2 \% \% \text {, Rote u. Zylinder. } \\
\text { Blut-RN } 65,24 \text { mg/dl. }\end{array}$ \\
\hline $\begin{array}{l}7,27 \\
7,31 \\
7,08\end{array}$ & $\begin{array}{l}+0,5 \\
-2,6\end{array}$ & $\begin{array}{l}226 \\
220 \\
210\end{array}$ & $\begin{array}{r}-2,6 \\
-\quad 7,1\end{array}$ & $\begin{array}{l}31,1 \\
30,1 \\
29,7\end{array}$ & $\begin{array}{l}-3,2 \\
-4,5\end{array}$ & $\begin{array}{l}0,565 \\
0,565 \\
0,565\end{array}$ & 160 & $\begin{array}{l}\text { Sekundäre Schrumpfniere. Odem an Un- } \\
\text { terschenkel u. Gesicht. Retinitis } \\
\text { nephritica. } \\
\text { Harn: Eiweiss } 5 \% \%, \text { Rote u. Zylinder } \\
\text { mässig. Blut-RN } 30,38 \mathrm{mg} / \mathrm{dl} \text {. } \\
\end{array}$ \\
\hline $\begin{array}{l}7,18 \\
7,24 \\
6,94\end{array}$ & $\begin{array}{l}+0,8 \\
-3,3\end{array}$ & $\begin{array}{l}296 \\
286 \\
277\end{array}$ & $\begin{array}{l}-3,4 \\
-6,4\end{array}$ & $\begin{array}{l}41,2 \\
39,5 \\
39,9\end{array}$ & $\begin{array}{l}-4,1 \\
-3,1\end{array}$ & $\begin{array}{l}0,595 \\
0,585 \\
0,590\end{array}$ & 220 & $\begin{array}{l}\text { Sckundäre Schrumpfnierc. Odeme an Bcin } \\
\text { kommen u. gehen. Retinitis nephritica. } \\
\text { Harn: Eitweiss 4\% \%os, Rote u. Zylinder. } \\
\text { Blut-RN 40,9 mg/dl. }\end{array}$ \\
\hline
\end{tabular}

zufuhr bleibt das Blut nämlich in seiner Konzentration entweder fast unverändert oder befindet sich in einem Zustand geringer Eindickung. Nach Ergebnissen vorliegenden Versuchs ist die Entscheidung darüber schwer zu treffen, ob das Blut erst nach vorausgegangener initieller Hydrämie in die primäre Eindickung umschlägt, oder ob es von vornherein, ohne jegliche Verdünnung erfahren zu haben, in primäre Eindickung eingeht.

In einschlägiger Literatur sind die Ansichten von verschiedenen Autoren nicht immer einig. $\mathrm{Marx}^{27)}$ ist z. B. der Meinung, dass bei Erkrankungen wie der Schumpfniere die initielle Hydrämie ganz leicht 
Fig. 8. Veränderungen des Serumeiweisses und dessen kolloid-osmotisehen Drucks durch orale Wasserzufuhr (Wasserversuch nach Volhard) bei maligner Hypertonie (Fall 1).

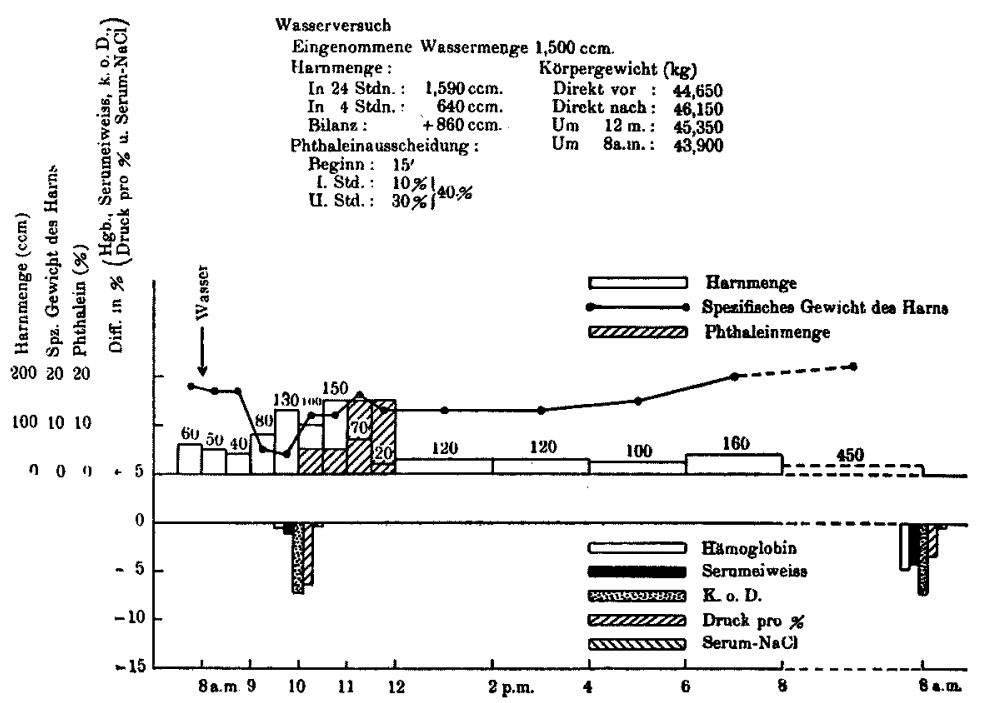

und verübergehend sei. Si e be $\mathrm{ck}^{29)}$ machte die Beobachtung, dass bei derart hochgradiger Niereninsuffizienz wie der Schrumpfniere die Blutverdünnung eher vermisst wird. Auch $\mathrm{N}$ onn enbruch ${ }^{30)}$ vertritt den Standpunkt, dass bei schwerer Niereninsuffizienz das Blut sich 2 und 4 Stunden nach der Wasserzufuhr in einem konzentrierten Zustand befinde. Weil es indessen bei meinen Untersuchungen weniger auf diese Fragestellung, als vielmehr auf den Einblick in die Verhältnisse des Eiweissaustausches ankommt, werde ich darauf nicht weiter eingehen, es möge die oben gestreifte Fragestellung künftigen Untersuchungen vorbehalten bleiben.

Eiweiss wird in Fällen, wo das Hämoglobin zunimmt, in wenn auch gewissem Ausmass vermehrt gefunden, und von 3 Fällen, bei denen das Hämoglobin annähernd konstant bleibt, nimmt das Eiweiss bei einem Fall erheblich zu und in 2 Fällen um ein Geringes ab. Es besteht also immerhin die Divergenz zwischen der Veränderung des Eiweisses und der des Hämoglobins. K. o.D. weist unter allen Umständen unfehlbar erhebliche Herabsetzung auf, ebenfalls der Druck pro \%.

29) Si e beck, Dtsch. Arch. f. klin. Med, 1919, 128, 173.

30) Nonnenbruch, Ibid., 1921, 136, 170. 
Fig. 9. Veränderungen des Serumeiweisses und dessen kolloid-osmotischen Drucks durch orale Wasserzufuhr (Wasserversuch nach Volhard) bei sekundärer Schrumpfniere (Fall 7).

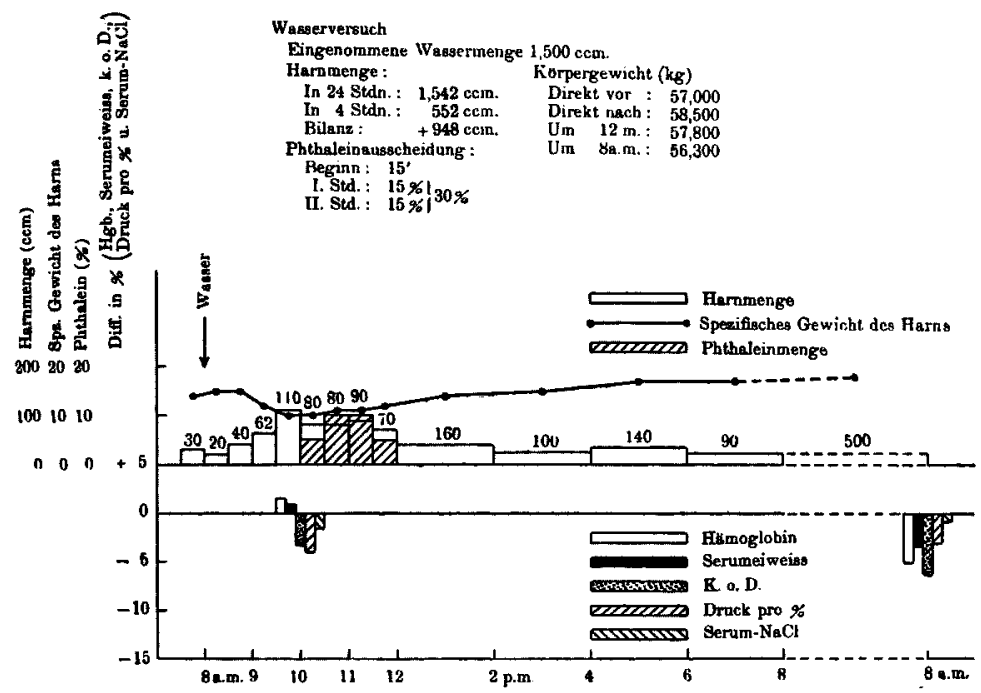

Wenn man einzelne Werte von Hämoglobin, Eiweiss und k. o. D. gegeneinander vergleichend betrachtet, so kann man ersehen, dass von sämtlichen 7 Fällen, ausgenornmen der Fall 3, in 6 Fällen das Eiweiss niedrigere Werte als das Hämoglobin aufweist, und dass der k. o. D. absinkt, wobei auch der Druck pro \% sich auffallend erniedrigt. In eben erwähnten Fällen muss also der Eiweissbestand des Blutes zum Teil ins Gewebe eingedrungen sein, und da diese extravasal ausgetretenen Eiweissteilchen feindisperser Natur gewesen sein müssen, so kommt es zu Erniedrigungen des k.o.D. sowie des Drucks pro \%. In einem restlichen Fall (Fall 3) vermehrt sich das Eiweiss, unabhängig vom Konstantbleiben des Hämoglobinwertes, was höchstwahrscheinlich durch die Eiweissmobilisation aus dem Depot begingt sein dürfte; aus dem Umstand indessen, dass der k. o.D. im Gegensatz zum Eiweiss eher absinkt und, dass auch der Druck pro \% erhebliche Abnahme erfährt, können Rückschlüsse darauf gezogen werden, dass hierbei mobilisierte Eiweissteilchen sicherlich grobdisperser Natur sind.

Bemerkenswert ist allerdings folgende Tatsache: Während näınlich bei bisher untersuchten Gesunden, Hypertonikem und Glomerulonephritiden nach der Flüssigkeitszufuhr der Übertritt des Bluteiweisses ins Gewebe und die Eiweissmobilisation aus dem Depotorgan, in wenn auch verschiedenen Graden, immer nebeneinander stattfinden, 
vollzieht sich bei Fällen von schwerer Niereninsuffizienz wie maligner Hypertonie und sekundärer Schrumpfniere in der Hauptsache das Hineindringen der Eiweissteilchen ins Gewebe, es sei denn, dass selten und zwar ausnahmsweise (nur in einem einzigen Fall unter 7 Fällen) die Eiweissmobilisation aus dem Depot überwiegt.

Wie aus dem Vergleich einzelner Daten über den Druck pro \% in oben beigegebenen Tabellen hervorgeht, ist die Erniedrigung des Drucks pro $\%$ bei Fällen von Schrumpfniere höchstgradig. Wenn man nun probeweise an Hand obiger Tabellen einzelne Daten über die prozentuelle Abnahme des Drucks pro \% in 2stündigem Ablauf gegeneinander vergleicht, so ist einleuchtend: Die prozentuelle Abnahme des Drucks pro \% beträgt nämlich bei Gesunden im Maximum 4,1 und im Minimum 0,4, bei Hypertonikern im Maximum 4,7, im Minimum 0,4 , ähnlich wie bei Gesunden, bei Glomerulonephritiden im Maximum 6,1, in Minimum 1,8. Bei Fällen von sekundärer Schrumpfniere und maligner Hypertonie beläuft sich die prozentuelle Abnahme ja im Maximum auf 7,8, im Mirimum auf 3,1. Als ein Beispiel hierfuir sind einschlägige Daten bei einem Fall von maligner Hypertonie (Fall 1) sowie bei einem Fall von sekundärer Schrumpfniere (Fall 7) in Figg. 8 und 9 aufgezeichnet.

Nach 24 Stunden ist der Hämoglobingehalt in allen Fällen ausnahmslos herabgesetzt, was auf das Zustandekommen der Blutverdünnung olne den Eintritt terminaler Bluteindickung hindeutet. Es ist dies, wie bereits von Daniel u. Högler, ${ }^{1)}$ Nonnenbruch ${ }^{30)}$ u. a. nachgewiesen worden ist, darauf zurückzuführen, dass beim Vorhandensein hochgradiger Niereninsuffizienz Wasser und andere Stoffe wohl anfänglich ins Gewebe übergehen können, welches aber bald in einen gesättigten Zustand versetzt wird, so dass im Blut der hydrämische Zustand längere Zeit fortbesteht. In dieser Periode zeigt das Bluteiweiss in sämtlichen Fällen die Tendenz zur Abnahme; der k. o. D. sowie Druck pro \% zeigen in der Mehrzahl der Fälle entweder, mit dem Eiweiss gleichen Schritt haltend, gewissermassen Erniedrigungen oder bleiben konstant. In der Minderzahl der Fälle bleibt der k. o. D. beim Auftreten der Eiweissabnahme konstant oder erfährt höchstens ein geringes Absinken, wodurch eine leichte Steigerung des Drucks pro \% verursacht wird. Im ganzen genommen jedoch zeigen das Bluteiweiss und der k. o. D. in 2stündigem Ablauf sowohl quantitativ wie auch qualitativ keine nennenswerten Veränderungen. Dies dürfte wahrscheinlich darauf beruhen, dass nach 2stündigem Ablauf der Austauschvorgang des Bluteiweisses zwischen Depot oder Gewebe an In- 
tensität abgenommen hat. Der Serum-NaCl-Spiegel erfährt durch Wasserzufuhr keine wesentliche Schwankung.

\section{Zusammenfassung.}

An Gesunden sowie an Fällen von essentieller Hypertonie, von Glomerulonephritis, sekundärer Schrumphniere und maligner Hypertonie wurde nach dem Verfahren des Volhard'schen Wasserversuchs die perorale Wasserzufuhr von $1,500 \mathrm{ccm}$ vorgenommen und unmittelbar vor der Flüssigkeitszufuhr sowie 2 und 24 Stunden später, im ganzen also dreimal Blutproben entnommen. An diesen Blutproben wurde durch Bestimmungen des Hämoglobingehaltes die Konzentrationsveränderung des Blutes, durch die Bestimmung des Kochsalzspiegels die Schwankungen der Krystalloide ermessen; es wurden weiterhin die Konzentration des Bluteiweisses sowie der kolloid-osmotische Druck bestimmt, um die nach peroraler Zufuhr grosser Flüsigkeitsmenge auftretende quantitativen und qualitativen Veränderungen des Bluteiweisses zu analysieren.

1. Bei Gesunden, bei denen die Wasserausscheidung, das Verdünnungs- und Konzentrationsvermögen und die Phthaleinausscheidung sich als vollkommen normal erweisen, befindet sich das Blut in 2stündigem Ablauf nach Wasserzufuhr schon in der Mehrzahl der Fälle nach vorausgegangener initieller Hydrämie in der Phase primärer Eindikkung, indem hier sich die Hämoglobinkonzentration vermehrt. Zur selben Stunde kommt es einerseits zu teilweisem Hineindringen kleinerer Eiweissteilchen vom Bluteiweissbestand ins Gewebe, während sich andererseits die Mobilisation grösserer Eiweissteilchen aus den Depotorganen vollzieht. Je nach jeweiligen Wechselbeziehungen der beiden Vorgänge erfahren die Bluteinweisskörper sowie der kolloidosmotische Druck bald eine Steigerung, bald eine Erniedrigung; der Druck pro \% sinkt, ganz davon unabhängig, stets ab.

Nach 24 Stunden finden sich neben terminaler Bluteindickung im allgemeinen Erhöhungen des Bluteiweisses, des kolloid-osmotischen Drucks und Druck pro \%. Bei gesunden Menschen äussern sich also die nach peroraler Wasserzufuhr auftretenden Veränderungen der Bluteiweisskörper in 2-stündigem Ablauf im regen Eiweissaustausch zwischen Blut und Geweben, während die Veränderungen in 24-stündigem Ablauf vorwiegend in einer einfachen Bluteindickung sich repräsentieren.

2. Bei Fällen von essentieller Hypertonie, in denen das Verdün- 
nungs- und Konzentrationsvermögen sowie die Phthaleinausscheidung sich als annähernd normal erweisen, und in welchen doch eine leichte Verzögerung der Wasserausscheidung anzutreffen ist, befindet sich das Blut 2 Stunden nach der Flüssigkeitszufuhr entweder noch in der Phase initieller Hydrämie oder im Übergangszustand zu primärer Eindickung. In dieser Zeitspanne spielt sich der Vorgang des Eiweissaustausches im grossen ganzen in analoger Weise ab, wie bei gesunden Individuen. In der Phase terminaler Bluteindickung in 24-stündigem Ablauf findet der Eiweissaustausch zwischen Depot und Gewebe noch, wenn auch in beschränkter Masse statt.

3. Bei Fällen von chronischer Glomeurlonephritis mit unerheblicher Niereninsuffizienz wird 2Stunden nach Flüssigkeitszufuhr in allen Fällen eine initielle Hydrämie befunden. DieBluteiweisskörper erfahren hierbei eine Abnahme, diese Abnahme ist aber durchaus nicht etwa durch eine Blutverdünnung bedingt, sondern sie kommt dadurch zustande, dass kleinere Eiweissteilchen vom Bluteiweissbestand zum Teil ins Gewebe eingedrungen ist, während zugleich grössere Eiweissteilchen im Depot in geringerem Masse ins Blut iibergetreten sind. Mithin erfahren der kolloid-osmotische Druck sowie der Druck pro \% gemeinsame Erniedrigungen. Nach 24 Stunden besteht der Austauschvorgang der Eiweisskörper zwischen Blut und Depot sowie Gewebe in noch intensiverem Grad fort, als bei Fällen von der essentiellen Hypertonie.

4. Bei Fällen von sekundärer Schrumpfniere und malignerHypertonie, in welch beiden die Wasserausscheidung, das Verdünnungsund Konzentrationsvermögen sowie die Phthaleinausscheidung hochgradig gestört sind, verlieren die Bluteiweisskörper 2 Stunden nach Flüssigkeitszufuhr vornehmlich an feindispersen Eiweissteilchen ins Gewebe, wobei der kolloid-osmotische Druck und Druck pro \% erhebliche Erniedrigungen erfahren. Die hierbei erfolgte prozentuelle Herabsetzung des Drucks pro \% ist grösser als bei Fällen von allen anderen Erkrankungen. In 24-stündigem Ablauf verharren die Bluteiweisskörper im ganzen genommen in denselben Veränderungen wie die in 2-stündigem Ablauf. Dies deutet offenbar darauf hin, dass der Eiweissaustausch zwischen Blut und Depot sowie Gewebe äusserst träge vor sich geht.

Was die Erniedrigung des Drucks pro \%, welche 2 Stunden nach Flüssigkeitszufuhr in Erscheinung tritt, anbetrifft, so ist sie bei maligner Hypertonie und sekundärer Schrumpfniere am grössten, bei chronischer Glomerulonephritis ohne Niereninsuffizienz weniger gross und 
endlich bei Fällen von essentieller Hypertonie sowie bei Gesunden in gemeinsam beinahe gleichem Masse leichter als bei letztgenannter Erkrankung, also am geringsten. Es dürfte dies, wie verschiedene Autoren (Cohnheim u. Lichtheim, ${ }^{31)}$ Magnus, ${ }^{32)}$ Nonnenbruch, ${ }^{33)}$ Schittenhelm u. Schlecht ${ }^{34)}$ u. a.) erkannt haben, von der Permeabilitätsänderung der Kapillarwand sowie von den physikalischchemischen Veränderungen an Geweben abhängig sein.

Die Veränderungen in 24-stündigem Ablauf äussern sich bei Gesunden in einer einfachen Bluteindickung, bei Fällen von essentieller Hypertonie und chronischer Glomerulonephritis besteht noch zur selben Stunde der Eiweissaustauschvorgang zwischen Gewebe und Blut in gewissem Ausmass fort, während dieser Vorgang bei maligner Hypertonie und sekundärer Schrumpfniere äusserst träge stattfindet.

31) Cohnheim u. Lichtheim, Virchow's Arch,, 1877, 69, 106.

32) Magnus, Arch. f. exp. Path. u. Pharmak., 1899, 42, 250.

33) Nonnen brueh, Dtsch. Arch. f. klin. Med., 1917, 122, 389.

34) Schittenhelm u. Schlecht, Die Ödemkrankheit, Berlin 1919, 82. 